\title{
Selection Theorem for Systems With Inheritance
}

\author{
A. N. Gorban ${ }^{1}$ \\ University of Leicester, LE1 7RH Leicester, UK
}

\begin{abstract}
The problem of finite-dimensional asymptotics of infinite-dimensional dynamic systems is studied. A non-linear kinetic system with conservation of supports for distributions has generically finite-dimensional asymptotics. Such systems are apparent in many areas of biology, physics (the theory of parametric wave interaction), chemistry and economics. This conservation of support has a biological interpretation: inheritance. The finite-dimensional asymptotics demonstrates effects of "natural" selection. Estimations of the asymptotic dimension are presented. After some initial time, solution of a kinetic equation with conservation of support becomes a finite set of narrow peaks that become increasingly narrow over time and move increasingly slowly. It is possible that these peaks do not tend to fixed positions, and the path covered tends to infinity as $t \rightarrow \infty$. The drift equations for peak motion are obtained. Various types of distribution stability are studied: internal stability (stability with respect to perturbations that do not extend the support), external stability or uninvadability (stability with respect to strongly small perturbations that extend the support), and stable realizability (stability with respect to small shifts and extensions of the density peaks). Models of self-synchronization of cell division are studied, as an example of selection in systems with additional symmetry. Appropriate construction of the notion of typicalness in infinite-dimensional space is discussed, and the notion of "completely thin" sets is introduced.
\end{abstract}

Key words: dynamics, attractor, dimension, evolution, entropy, natural selection AMS subject classification: 92D15, 37L30, 54H20

\footnotetext{
${ }^{1}$ E-mail: ag153@le.ac.uk
} 


\section{Introduction: Unusual Conservation Law}

There are three geometrically distinguished classes of dynamical systems originating from natural sciences:

- Hamiltonian systems;

- Dissipative systems with entropy (or another thermodynamic Lyapunov function); and

- Systems with inheritance.

Hamiltonian systems originated from mechanics. Symplectic geometry followed them. Dissipative systems with thermodynamic Lyapunov functions arose from thermodynamics and kinetics. The related geometry is the geometry of Legendre transformation and contact structures (this subject is not exhausted yet; see for example [85, 29] and a recent publication [37]). Systems with inheritance are emerging from population dynamics, physical kinetics, turbulence theory, and many other fields of natural science. The geometrical sense of inheritance is a special conservation law, in which the conserved "quantity" is a set, a support of distribution.

In the 1970s to the 1980s, theoretical work developed another "common" field simultaneously applicable to physics, biology and mathematics. For physics it is (so far) part of the theory of a special kind of approximation, demonstrating, in particular, interesting mechanisms of discreteness in the course of the evolution of distributions with initially smooth densities. However, what for physics is merely a convenient approximation is a fundamental law in biology: inheritance. The consequences of inheritance (collected in the selection theory $[15,39,58,76,77,2,22,41,29,32,73]$ ) give one of the most important tools for biological reasoning.

This paper is not a review of the scientific literature on evolution, and we mention here only references that are particularly important for our understanding of the selection theory and its applications.

Consider a community of animals. Let it be biologically isolated. Mutations can be neglected in the first approximation. In this case, new genes do not emerge.

An example from physics is as follows. Let waves with wave vectors $k$ be excited in some system. Denote $K$ a set of wave vectors $k$ of excited waves. Let the wave interaction does not lead to the generation of waves with new $k \notin K$. Such an approximation is applicable to a variety of situations, and has been described in detail for wave turbulence in [86, 87].

What is common in these examples is the evolution of a distribution with a support that does not increase over time.

What does not increase must, as a rule, decrease, if the decrease is not prohibited. This naive thesis can be converted into rigorous theorems for the case under consideration [29]. It is proved that the support decreases in the limit $t \rightarrow \infty$ if it was sufficiently large initially. (At finite times the distribution supports are conserved and decrease only in the limit $t \rightarrow \infty$.) Conservation of the support usually results in the following effect: dynamics of an initially infinite-dimensional system at $t \rightarrow \infty$ can be described by finite-dimensional systems. 
The simplest and most common class of equations in applications for which the distribution support does not grow over time is constructed as follows. To each distribution $\mu$ is assigned a function $k_{\mu}$ by which distributions can be multiplied. Let us write down the equation:

$$
\frac{\mathrm{d} \mu}{\mathrm{d} t}=k_{\mu} \times \mu .
$$

The multiplier $k_{\mu}$ is called a reproduction coefficient. It depends on $\mu$, and this dependence can be rather general and non-linear.

Two remarks can be important:

1. The apparently simple form of (1.1) does not mean that this system is linear or even close to linear. The operator $\mu \mapsto k_{\mu}$ is a general non-linear operator, and the only restriction is its continuity in an appropriate sense (see below).

2. On a finite set $X=\left\{x_{1}, \ldots, x_{n}\right\}$, non-negative measures $\mu$ are simply non-negative vectors $\mu_{i} \geq 0(\mathrm{i}=1, \ldots, \mathrm{n})$, and (1.1) appears to be a system of equations of the following type:

$$
\frac{\mathrm{d} \mu_{i}}{\mathrm{~d} t}=k_{i}\left(\mu_{1}, \ldots, \mu_{n}\right) \times \mu_{i},
$$

and the only difference from a general dynamic system is the special behavior of the right-hand side of (1.2) near zero values of $\mu_{i}$.

The right-hand side of (1.1) is the product of the function $k_{\mu}$ and the distribution $\mu$, and hence $\mathrm{d} \mu / \mathrm{d} t$ should be zero when $\mu$ is equal to zero; therefore the support of $\mu$ is conserved in time (over finite times).

Let us start a more formal consideration, with basic definitions and notations. First, we introduce the space of inherited units $X$. In this paper $X$ is a compact metric space with a metric $\rho(x, y)$. In other special sections we assume that $X$ is a closed bounded domain in finite-dimensional real space $R^{n}$. As a particular case of compact space, a finite set $X$ can be discussed.

We study the dynamics of distributions on $X$. Each distribution on a compact space $X$ is a continuous linear functional on the space of continuous real functions $C(X)$. We follow the Bourbaki approach [10]: a measure is a continuous functional, an integral. Bourbaki's book [10] contains all the necessary notions and theorems (and much more material than we need here). Space $C(X)$ is a Banach space endowed with the maximum norm

$$
\|f\|=\max _{x \in X}|f(x)| \text {. }
$$

If $\mu \in C^{*}(X)$ is a continuous function and $f \in C(X)$, then $[\mu, f]$ is the value of $\mu$ at a function $f$.

Let us mention here two other notations. If $X$ is a bounded closed subset of a finitedimensional space $R^{n}$, then we represent this functional as the integral

$$
[\mu, f]=\int \mu(x) f(x) \mathrm{d} x,
$$


which is the standard notation for distribution (or generalized function) theory. Note that here the "density" $\mu(x)$ is not assumed to be an absolute continuous function with respect to the Lebesgue measure $\mathrm{d} x$ (or even a function), and the notation in Eq. (1.4) has the same sense as $[\mu, f]$. If the measure is defined as a function on a $\sigma$-algebra of sets, then the following notation is used:

$$
[\mu, f]=\int f(x) \mu(\mathrm{d} x) .
$$

We use the notation $[\mu, f]$ for general spaces $X$ and the representation (1.4) on domains in $R^{n}$ without any additional comments. The product $k \times \mu$ is defined for any $k \in C(X)$, $\mu \in C^{*}(X)$ by the equality: $[k \mu, f]=[\mu, k f]$.

The support of $\mu, \operatorname{supp} \mu$, is the smallest closed subset of $X$ with the following property: if $f(x)=0$ on $\operatorname{supp} \mu$, then $[\mu, f]=0$, i.e. $\mu(x)=0$ outside $\operatorname{supp} \mu$.

In the space of measures we use weak $k^{*}$ convergence, i.e. the convergence of averages:

$$
\mu_{i} \rightarrow \mu^{*} \text { if and only if }\left[\mu_{i}, \varphi\right] \rightarrow\left[\mu^{*}, \varphi\right]
$$

for all continuous functions $\varphi \in C(X)$. This weak* convergence of measures generates weak topology on the space of measures (sometimes called weak topology of conjugated space, or wide topology).

Strong topology on the space of measures $C^{*}(X)$ is defined by the norm $\|\mu\|=\sup _{\|f\|=1}[\mu, f]$.

Strictly speaking, the space on which $\mu$ is defined and the distribution class it belongs to should be specified. The properties of the mapping $\mu \mapsto k_{\mu}$ should also be specified, and the existence and uniqueness of solutions of (1.1) under given initial conditions should be identified. In specific situations the answers to these questions are not difficult.

The sequence of continuous functions $k_{i}(x)$ is considered to be convergent if it converges uniformly. The sequence of measures $\mu_{i}$ is called convergent if for any continuous function $\varphi$ the integrals $\left[\mu_{i}, \varphi\right]$ converge [weak* convergence (1.5)]. The mapping $\mu \mapsto k_{\mu}$ assigning the reproduction coefficient $k_{\mu}$ to the measure $\mu$ is assumed to be continuous with respect to these convergencies.

Finally, the space of measures is assumed to have a bounded set $M$ that is positively invariant relative to system (1.1): if $\mu(0) \in M$, then $\mu(t) \in M$ (we also assume that $M$ is non-trivial, i.e. it is neither empty nor a one-point set). This $M$ serves as the phase space of system (1.1). (Let us remind that the set of measures $M$ is bounded if the set of integrals $\{[\mu, f] \mid \mu \in M,\|f\| \leq 1\}$ is bounded, where $\|f\|$ is the norm (1.3).) We study dynamic of system (1.1) in bounded positively invariant set $M$.

Most of the results for systems with inheritance use a theorem on weak* compactness: The bounded set of measures is precompact with respect to weak* convergence (i.e. its closure is compact). Therefore the set of corresponding reproduction coefficients $k_{M}=\left\{k_{\mu} \mid \mu \in M\right\}$ is precompact.

Let us start with the simplest example and the first theorem, and then discuss possible interpretations. The simplest example of an emerging discrete distribution from a continuous 
initial distribution gives us the following equation:

$$
\frac{\partial \mu(x, t)}{\partial t}=\left[f_{0}(x)-\int_{a}^{b} f_{1}(x) \mu(x, t) \mathrm{d} x\right] \mu(x, t),
$$

where the functions $f_{0}(x)$ and $f_{1}(x)$ are positive and continuous on the closed segment $[a, b]$. Let the function $f_{0}(x)$ reach the global maximum on the segment $[a, b]$ at a single point $x_{0}$. If $x_{0} \in \operatorname{supp} \mu(x, 0)$, then:

$$
\mu(x, t) \rightarrow \frac{f_{0}\left(x_{0}\right)}{f_{1}\left(x_{0}\right)} \delta\left(x-x_{0}\right), \quad \text { when } t \rightarrow \infty,
$$

where $\delta\left(x-x_{0}\right)$ is the $\delta$-function.

If $f_{0}(x)$ has several global maxima, then the right-hand side of (1.7) can be the sum of a finite number of $\delta$-functions. Here a natural question arises: is it worth considering such a possibility? Should not we deem it improbable for $f_{0}(x)$ to have more than one global maximum? Indeed, such a case seems to be very unlikely to occur. More details on this are given below.

The limit behavior of a typical system with inheritance (1.1) can be much more complicated than (1.7). Here we can mention that any finite-dimensional system with a compact phase space can be embedded in a system with inheritance (1.2). An additional possibility for the limit behavior is, for example, the drift effect (Section 3.1).

The first step in the routine investigation of a dynamical system is a question about fixed points and their stability. The first observation concerning the system (1.1) is that it can only be asymptotically stable for steady-state distributions, the support of which is discrete (i.e. the sums of $\delta$-functions). This can be proved for all consistent formalizations. Thus, we have the first theorem.

Theorem 1.1. The support of asymptotically stable distributions for the system (1.1) is always discrete.

Proof. Let $U$ be a domain in $X$, and the "total amount" in $U$ (integral of $|\mu|$ over $U$ ) be less than $\varepsilon>0$ but not equal to zero. Let us substitute distribution $\mu$ by zero on $U$ (the rest remains as it is). It is natural to consider this disturbance of $\mu$ as $\varepsilon$-small. However, if the dynamics is described by (1.1), there is no way back to the undisturbed distribution, because the support cannot increase. If the steady-state distribution $\mu^{*}$ is asymptotically stable, then for some $\varepsilon>0$ any $\varepsilon$-small perturbation of $\mu^{*}$ relaxes back to $\mu^{*}$. This is possible only if for any domain $U$ the integral of $\left|\mu^{*}\right|$ over $U$ is either 0 or greater than $\varepsilon$. Hence, this asymptotically stable distribution $\mu^{*}$ is the sum of a finite number of point measures:

$$
\mu^{*}(x)=\sum_{i=1}^{q} N_{i} \delta_{x_{i}}
$$

with $\left|N_{i}\right|>\varepsilon$ for all $i$ and where $\delta_{x_{i}}$ is the normalized point measure at point $x_{i}$. In distribution theory notation, this corresponds to the $\delta$-function $\delta\left(x-x_{i}\right)$.

The perturbation discussed is small not only in the weak* topology, but also in the strong sense, and thus it is sufficient to consider strongly small perturbations to prove that 
the asymptotically stable distribution should be discrete. Hence, this statement is true if the operator $\mu \mapsto k_{\mu}$ is continuous for strong topology on the space of measures. This is a significantly weaker requirement than being continuous in weak* topology.

This simple observation has many strong generalizations to general $\omega$-limit points, to equations for vector measures, etc.

Dynamic systems in which the phase variable is a distribution $\mu$ and distribution support is the integral of motion frequently occur in both physics and biology. Because of their attractive properties, they are frequently used as approximations: we try to find the "main part" of the system in the form of (1.1), and represent the rest as a small perturbation of the main part.

Equations in the form of (1.1) allow the following biological interpretation: $\mu$ is the distribution of the number (or of biomass or another extensive variable) over inherited units: species, varieties, supergenes, genes. Whatever is considered as the inherited unit depends on the context and the specific problem. The value of $k_{\mu}(x)$ is the reproduction coefficient of the inherited unit $x$ under given conditions. The notion of "given conditions" includes the distribution $\mu$, and the reproduction coefficient depends on $\mu$. Equation (1.6) can be interpreted as follows: if $f_{0}(x)$ is the specific birth-rate of the inherited unit $x$ (below, for the sake of definiteness, $x$ is a variety, following the spirit of Darwin's seminal work [15]), the death rate for the representatives of all inherited units (varieties) is determined by one common factor depending on the density $\int_{a}^{b} f_{1}(x) \mu(x, t) \mathrm{d} x ; f_{1}(x)$ is the individual contribution of variety $x$ to this death rate.

On the other hand, for systems of waves with a parametric interaction, $k_{\mu}(x)$ can be the amplification (decay) rate of the wave with wave vector $x$.

Conservation of the support in (1.1) can be considered as inheritance, and we call system (1.1) and its nearest generalizations "systems with inheritance". Traditional separation of the process of transferring biological information into inheritance and mutations, which are small in any admissible sense, can be compared to a description according to the following pattern: system (1.1) (or its nearest generalizations) plus small disturbances. Beyond the limits of such a description, discussion of inheritance loses the conventional sense.

In biology such an approximation is essentially applicable to all classical genetics, and to the formal content of the theory of natural selection. The initial diversity is "thinned out" over time, and the limit distribution supports are described by some extremal principles (principles of optimality).

The first study of dynamics systems with inheritance was carried out by J. B. S. Haldane. He used the simplest examples, studied steady-state distributions and obtained the extremal principle for them. His pioneering book "The Causes of Evolution" (1932) [39] gives a clear explanation of the connections between inheritance (the conservation of distribution support) and the optimality of selected varieties.

Haldane's work was followed by an entirely independent series of works on the S-approximation in the spin wave theory and on wave turbulence $[86,87,55]$, which studied wave configurations in the approximation of an "inherited" wave vector, and by "synergetics" [40], in which the "natural selection" of modes is one of the basic concepts. 
At the same time, a series of works on biological kinetics was completed (see, for example $[70,27,73,29])$. These studies addressed not only steady states, but also general limit distributions [27, 29] and waves in the space of inherited units [70]. For steady states a new type of stability was described - stable realizability (see below). Many examples of ecological applications are collected in reference [73]. The application of optimality principles to crop growth simulation is analyzed in reference [66]. Some attempts using sociological applications are also known [75].

The Haldane achievements were continued by works on stable evolutionary strategies and evolutionary games. In works by Maynard Smith [76, 77] the "War of Attrition" model of animal conflict was introduced and the notion of evolutionarily stable strategy (ESS) was defined. This notion was elaborated further in many papers $[79,44,80,82,14,9,4,5,8,6$, $7,62,63,21]$. The reader is also referred to a recent review [45], in which evolutionary game dynamics is defined as the application of population dynamical methods to game theory. (It was invented by evolutionary biologists, but had a great impact on modern game theory and economical mathematics.) For some classes of models (a "generalized war of attrition" [2]), it was demonstrated that either (i) there is no ESS or (ii) there is a unique ESS, which is fully specified. In the case for which only a finite number of pure strategies is available, global convergence to the ESS is shown. Of course, for systems with inheritance (1.1), more complicated behavior is also possible. In reference [82] collections of subsets that might be supports for ESSs were identified. Imaginary experiments with mutant invasion are based on the notion of EES [76, 77]. The dynamical foundation of this notion and a dynamical theory of uninvadability in the context of stability theory have been developed [4]. It should be mentioned that the analogue of uninvadability, external stability, was one of the main notions of the $S$-theory in physics [86, 87, 55], and ecological applications of this notion were developed reasonably far in the 1970s-1980s; see references [29, 32, 73] and references therein.

The dynamics of evolutionary games for the case of a continuum of possible strategies has been investigated [8]. The stability properties of stationary points were studied and some examples were given. In fact, in reference [8] a particular case of systems with inheritance was studied; in this case $X$ is the space of strategies of an evolutionary game.

The setwise notions of stable evolutionary sets were introduced [80] for evolutionary game models in which there is a continuum of equilibrium states, with no state stable in itself, but which together are evolutionarily stable. This concept was also analyzed from a dynamical point of view [6].

Recently, the theory of evolutionary games with a continuum of possible strategies has been developed very intensively [7, 62, 63, 21]; see also the review in reference [45].

The first biological applications of systems with inheritance were population dynamics and mathematical genetics. All the classical equations for distributions of animals or genes have the form (1.1) (or a generalization with additional variables). The space of inherited units, $X$, in these cases has a direct biological interpretation: it is the space of inherited variations, or species, or the space of alleles ("variations of genes"). In ecological applications it has been proved [73] that the concept of inherited variations of animals (without consid- 
eration of alleles) gives appropriate accuracy in the problem of succession, that is, in the modeling and simulation of changes in a biological community under changing conditions. But what is gene? Biology returns to this question again and again [25]. The interplay between "units of function" and "units of inheritance" for different time scale produces very complicated and fascinating picture.

Epigenetic inherited units yield many interesting materials for modeling. The source of dynamical difference between genetic and epigenetic inheritance is their different time scales when they are different [67]. The interaction between these types of inheritance could be quite mysterious. For example, the rates and some specific properties of genetic mutation processes might be inherited properties, as was discovered for the effect of transgenerational instability [20]. This phenomenon is probably due to an epigenetic mechanism.

For the "ecological time scale", the maternity effect forms another group of inherited units. These units are important for the evolution at ecological time-scales [68].

The space of inherited strategies provides the interpretation of $X$ in many applications. In particular, the selection of strategies of the spatial distribution of individuals has been studied [35]. In the case of non-monotonous dependence of the reproduction coefficient on the mean population density, a cluster formation was proven. This theory was applied to an investigation of the creation of cellular clusters in flow-rate cultivators [36].

It is clear that animal migration is not completely random and that it depends on conditions; in particular, predator migration depends on space variations of the prey density, which might imply interesting dynamical consequences, including changes in the number and stability of equilibria and limit cycles [46]. Models of evolutionarily optimal migration strategies in prey-predator systems have been studied [71]. A great variety of dynamic regimes has been observed, and some of them could be interpreted as outbreak explosions.

The distribution of successors over time (that represent variations of individual development, delayed maturation and even a pause in ontogenesis) is important in the adaptation to stochastic conditions. Evolutionarily optimal strategies of facultative diapause for forest insects have been studied [73]. In [81] a population with complicated dynamics was studied numerically. It was demonstrated that random noise shifts the system towards a higher probability of delaying maturation.

In ecological physiology, the points of $X$ represent strategies of physiological adaptation. A useful notion is the adaptation resource. The presentation of the adaptation process as a redistribution of this resource for the neutralization of external factors is an effective tool for adaptation modelling [34]. These models of "factors-resource" and the dynamic theory of optimal evolutionary strategies allowed us to develop "correlation adaptometry" [72, 33]. This method of comparative ecological physiology is now in use for comparative analysis of populations and groups for a wide range of organisms, from the human population of the Far North [65, 78] to herbaceous species [48].

The purpose of this paper is to present general results for the theory of systems with inheritance: optimality principles for limit distributions, theorems on selection, estimations of the limit diversity (estimates of a number of points in the support for limit distributions), the drift effect and drift equations. Some of these results have been published in preprint 
$[27,28]$ and, partially, book form [29].

The main benefit of the special form of systems with inheritance is the possibility of describing the limit behavior of such systems by avoiding the solution of equations. A system of weak and strong optimality principles describes the supports of limit or stable distributions. A special drift asymptotic reduces asymptotic behavior at large time values by a finite system of ordinary differential equations (ODEs). In subsequent sections this technique is developed on the basis of investigation of the general system represented by Equation (1.1).

The outline of the paper is as follows. In the next section the optimality principles for supports of $\omega$-limit distributions are developed. These principles have a "weak" form; the set of possible supports is estimated from above and it is not obvious that this estimation is effective (this is proved in Section 2.4).

Minimax estimations of the number of points in the support of $\omega$-limit distributions are given in Section 2.2. The idea is to study systems under a $\varepsilon$-small perturbation, to estimate the maximal number of points for each realization of the perturbed system, and then to estimate the minimum of these maxima among various realizations. These minimax estimates can be constructive and do not use integration of the system. The set of reproduction coefficients $\{k(\mu) \mid \mu \in M\}$ is compact in $C(X)$. Therefore, this set can be approximated by a finite-dimensional linear space $L_{\varepsilon}$ with any given accuracy $\varepsilon$.

The number of coexisting inherited units ("quasi-species") is estimated from above as $\operatorname{dim} L_{\varepsilon}$. This estimate is true both for stationary and non-stationary coexistence. In its general form this estimate was proved in 1980 [27, 29], but the reasoning of this type has a long history. Perhaps, G. Gause [26] was the first to suggest the direct connection between the number of species and the number of resources. One can call this number "dimension of the environment." He proposed the famous concurrent exclusion principle. This principle is often named as the Gause principle, but G. Gause considered his work as development of Ch. Darwin ideas of the struggle for existence. This is obvious even from the title of his book [26]. More details about early history of the concurrent exclusion principle are presented in the review paper of G. Hardin [42].

MacArthur and Levins [56] suggested that the number of coexisting species is limited by the number of ecological resources. Later [57], they studied the continuous resource distribution (niche space) where the number of species is limited by the fact that the niches must not overlap too much. In 1999, G. Meszena and J.A.J. Metz [60] developed further the idea of environmental feedback dimensionality (perhaps, independently of $[27,29]$ ). In 2003 [17], the theory of structurally stable stationary coexistence was developed, and in 2006 the idea of robustness in concurrent exclusion was approached again, as a "unified theory" of "competitive exclusion and limiting similarity" [59]. All these achievements are related to estimation of dimension of the set $\{k(\mu) \mid \mu \in M\}$ or of some its subsets. This dimension plays the role of "robust dimension of population regulation".

Section 2.3 contains auxiliary results from functional analysis. Two problems are studied: (i) how to describe the sets of global maximum points in a typical compact set of continuous functions on a compact metric space; and (ii) how to define the "typicalness" in Banach 
spaces in order to meet intuitive expectations. It is obvious that typicalness in the sense of Baire category violates some of the essential requirements of common sense, for example even a real line can be divided into a set of the zero measure and a set of first category. Hence, a stronger notion is needed. Completely thin sets are introduced and the typical properties of compact sets of continuous functions are studied (the sets of exclusions are completely thin).

A theorem of selection efficiency is presented in Section 2.4. The sense of this theorem is as follows: for almost every system the support of all $\omega$-limit distributions is small (in an appropriate strong sense). Its geometrical interpretation suggested by M. Gromov is explained in Section 2.5. Specific entropy-like functions, the decreasing measures of diversity, are constructed in Section 2.6. Decreasing of these functions describes self-organization.

The drift equations (Section 3.1) describe the asymptotic behavior of systems with inheritance near the limit distributions (when $X$ is a domain in $R^{n}$, or a manifold). That asymptotics proves to be the motion of narrow, almost Gaussian peaks. The drift equations are ODEs. In fact, the drift equations substitute the initial infinite-dimensional dynamic system (1.1) in many applications: usually the system has enough time to reach the drift asymptotic. The bifurcations with change of number of peaks deserve special attention: this branching-type" evolution [19], can be related to speciation.

The simplest model for "reproduction + small mutations" is developed. The limit of zero mutations is singular, because arbitrary small (but non-zero) mutations added to equation (1.1) destroy dynamical invariance of subspaces $\{\mu \mid \operatorname{supp} \mu \subseteq Y\}$ for closed subsets $Y \subset X$. Nevertheless, if we consider initial distributions $\mu_{0}$ with $\operatorname{supp} \mu=X$ (all variability is actually present), then sufficiently small mutations change nearly nothing, just the limit $\delta$-shaped peaks transform into sufficiently narrow peaks, and zero limit of the velocity of their drift at $t \rightarrow \infty$ substitutes by a small finite one in the presence of drift effect. Moreover, there exists a scale invariance, and dynamics for large $t$ does not depend on nonzero mutation intensity, if the last is sufficiently small: to change this intensity, we need just to rescale time.

Various types of distribution stability are studied in Section 3.3: internal stability (stability with respect to perturbations that do not extend the support), external stability or uninvadability (stability to the strongly small perturbation which extend the support), and stable realizability (stability with respect to a small shift and small extension of density peaks). The general condition for stable realizability is the usual ODE Lyapunov stability condition with respect to the corresponding drift equations.

The cell population structured by age (and age-defined variables, size, chemical properties, etc.) is studied in Section 4. The most restrictive assumption in the model is that all cells have the same cell-cycle period, $T$. Hence, the cell-cycle phase is the inherited variable. Nevertheless, the general results from previous sections cannot be applied to this model because of additional symmetry. Direct analysis of the example shows that in this case selection is also efficient and the equivariant selection theory is possible. This selection is an example of self-synchronization. Small deviations from the basic assumption lead to smooth self-synchronization waves, and large deviations can destroy the effect.

In Section 5 a brief description of the main results is presented. 


\section{Selection Theorem}

\subsection{Optimality Principle for Limit Diversity}

Description of the limit behavior of a dynamical system can be much more complicated than enumerating stable fixed points and limit cycles. The leading rival to adequately formalize the limit behavior is the concept of the " $\omega$-limit set". It was discussed in detail in the classical monograph [1]. The fundamental textbook on dynamical systems [43] and the introductory review [49] are also available.

Let $f(t)$ be the dependence of the position of point in the phase space on time $t$ (i.e. the motion of the dynamical system). A point $y$ is a $\omega$-limit point of the motion $f(t)$, if there exists such a sequence of times $t_{i} \rightarrow \infty$, that $f\left(t_{i}\right) \rightarrow y$.

The set of all $\omega$-limit points for the given motion $f(t)$ is called the $\omega$-limit set. If, for example, $f(t)$ tends to the equilibrium point $y^{*}$ then the corresponding $\omega$-limit set consists of this equilibrium point. If $f(t)$ is winding onto a closed trajectory (the limit cycle), then the corresponding $\omega$-limit set consists of the points of the cycle and so on.

General $\omega$-limit sets are not encountered oft in specific situations. This is because of the lack of efficient methods to find them in a general situation. Systems with inheritance is a case, where there are efficient methods to estimate the limit sets from above. This is done by the optimality principle.

Let $\mu(t)$ be a solution of (1.1). Note that

$$
\mu(t)=\mu(0) \exp \int_{0}^{t} k_{\mu(\tau)} \mathrm{d} \tau .
$$

Here and below we do not display the dependence of distributions $\mu$ and of the reproduction coefficients $k$ on $x$ when it is not necessary. Fix the notation for the average value of $k_{\mu(\tau)}$ on the segment $[0, t]$

$$
\left\langle k_{\mu(t)}\right\rangle_{t}=\frac{1}{t} \int_{0}^{t} k_{\mu(\tau)} \mathrm{d} \tau
$$

Then the expression (2.8) can be rewritten as

$$
\mu(t)=\mu(0) \exp \left(t\left\langle k_{\mu(t)}\right\rangle_{t}\right) .
$$

If $\mu^{*}$ is the $\omega$-limit point of the solution $\mu(t)$, then there exists such a sequence of times $t_{i} \rightarrow \infty$, that $\mu\left(t_{i}\right) \rightarrow \mu^{*}$. Let it be possible to chose a convergent subsequence of the sequence of the average reproduction coefficients $\left\langle k_{\mu(t)}\right\rangle_{t}$, which corresponds to times $t_{i}$. We denote as $k^{*}$ the limit of this subsequence. Then, the following statement is valid: on the support of $\mu^{*}$ the function $k^{*}$ vanishes and on the support of $\mu(0)$ it is non-positive:

$$
\begin{aligned}
& k^{*}(x)=0 \text { if } x \in \operatorname{supp} \mu^{*}, \\
& k^{*}(x) \leq 0 \text { if } x \in \operatorname{supp} \mu(0) .
\end{aligned}
$$

Taking into account the fact that $\operatorname{supp} \mu^{*} \subseteq \operatorname{supp} \mu(0)$, we come to the formulation of the optimality principle (2.10): The support of limit distribution consists of points of the 
global maximum of the average reproduction coefficient on the initial distribution support. The corresponding maximum value is zero.

We should also note that not necessarily all points of maximum of $k^{*}$ on $\operatorname{supp} \mu(0)$ belong to $\operatorname{supp} \mu^{*}$, but all points of $\operatorname{supp} \mu^{*}$ are the points of maximum of $k^{*}$ on $\operatorname{supp} \mu(0)$.

If $\mu(t)$ tends to the fixed point $\mu^{*}$, then $\left\langle k_{\mu(t)}\right\rangle_{t} \rightarrow k_{\mu^{*}}$ as $t \rightarrow \infty$, and supp $\mu^{*}$ consists of the points of the global maximum of the corresponding reproduction coefficient $k_{\mu^{*}}$ on the support of $\mu^{*}$. The corresponding maximum value is zero.

If $\mu(t)$ tends to the limit cycle $\mu^{*}(t)\left(\mu^{*}(t+T)=\mu^{*}(t)\right)$, then all the distributions $\mu^{*}(t)$ have the same support. The points of this support are the points of maximum (global, zero) of the averaged over the cycle reproduction coefficient

$$
k^{*}=\left\langle k_{\mu^{*}(t)}\right\rangle_{T}=\frac{1}{T} \int_{0}^{T} k_{\mu^{*}(\tau)} \mathrm{d} \tau,
$$

on the support of $\mu(0)$.

The supports of the $\omega$-limit distributions are specified by the functions $k^{*}$. It is obvious where to get these functions from for the cases of fixed points and limit cycles. There are at least two questions: what ensures the existence of average reproduction coefficients at $t \rightarrow \infty$, and how to use the described extremal principle (and how efficient is it). The latter question is the subject to be considered in the following sections. In the situation to follow the answers to these questions have the validity of theorems.

Due to the theorem about weak ${ }^{*}$ compactness, the set of reproduction coefficients $k_{M}=$ $\left\{k_{\mu} \mid \mu \in M\right\}$ is precompact, hence, the set of averages (2.9) is precompact too, because it is the subset of the closed convex hull $\overline{\operatorname{conv}}\left(k_{M}\right)$ of the compact set. This compactness allows us to claim the existence of the average reproduction coefficient $k^{*}$ for the description of the $\omega$-limit distribution $\mu^{*}$ with the optimality principle (2.10).

\subsection{How Many Points Does the Limit Distribution Support Hold?}

The limit distribution is concentrated in the points of (zero) global maximum of the average reproduction coefficient. The average is taken along the solution, but the solution is not known beforehand. With the convergence towards a fixed point or to a limit cycle this difficulty can be circumvented. In the general case the extremal principle can be used without knowing the solution, in the following way [29]. Considered is a set of all dependencies $\mu(t)$ where $\mu$ belongs to the phase space, the bounded set $M$. The set of all averages over $t$ is $\left\{\left\langle k_{\mu(t)}\right\rangle_{t}\right\}$. Further, taken are all limits of sequences formed by these averages - the set of averages is closed. The result is the closed convex hull $\overline{\operatorname{conv}}\left(k_{M}\right)$ of the compact set $k_{M}$. This set involves all possible averages (2.9) and all their limits. In order to construct it, the true solution $\mu(t)$ is not needed.

The weak optimality principle is expressed as follows. Let $\mu(t)$ be a solution of (1.1) in $M, \mu^{*}$ is any of its $\omega$-limit distributions. Then in the set $\overline{\operatorname{conv}}\left(k_{M}\right)$ there is such a function 
$k^{*}$ that its maximum value on the support $\operatorname{supp} \mu_{0}$ of the initial distribution $\mu_{0}$ equals to zero, and $\operatorname{supp} \mu^{*}$ consists of the points of the global maximum of $k^{*}$ on supp $\mu_{0}$ only (2.10).

Of course, in the set $\overline{\operatorname{conv}}\left(k_{M}\right)$ there are usually many functions that are irrelevant to the time average reproduction coefficients for the given motion $\mu(t)$. Therefore, the weak extremal principle is really weak - it gives too many possible supports of $\mu^{*}$. However, even such a principle can help to obtain useful estimates of the number of points in the supports of $\omega$-limit distributions.

It is not difficult to suggest systems of the form (1.1), in which any set can be the limit distribution support. The simplest example: $k_{\mu} \equiv 0$. Here $\omega$-limit (fixed) is any distribution. However, almost any arbitrary small perturbation of the system destroys this pathological property.

In the realistic systems, especially in biology, the coefficients fluctuate and are never known exactly. Moreover, the models are in advance known to have a finite error which cannot be exterminated by the choice of the parameters values. This gives rise to an idea to consider not individual systems (1.1), but ensembles of similar systems [29].

Let us estimate the maximum for each individual system from the ensemble (in its $\omega$-limit distributions), and then, estimate the minimum of these maxima over the whole ensemble (the minimax estimation). The latter is motivated by the fact, that if the inherited unit has gone extinct under some conditions, it will not appear even under the change of conditions.

Let us consider an ensemble that is simply the $\varepsilon$-neighborhood of the given system (1.1). The minimax estimates of the number of points in the support of $\omega$-limit distribution are constructed by approximating the dependencies $k_{\mu}$ by finite sums

$$
k_{\mu}=\varphi_{0}(x)+\sum_{i=1}^{n} \varphi_{i}(x) \psi_{i}(\mu) .
$$

Here $\varphi_{i}$ depend on $x$ only, and $\psi_{i}$ depend on $\mu$ only. Let $\varepsilon_{n}>0$ be the distance from $k_{\mu}$ to the nearest sum (2.11) (the "distance" is understood in the suitable rigorous sense, which depends on the specific problem). So, we reduced the problem to the estimation of the diameters $\varepsilon_{n}>0$ of the set $\overline{\operatorname{conv}}\left(k_{M}\right)$.

The minimax estimation of the number of points in the limit distribution support gives the answer to the question, "How many points does the limit distribution support hold": If $\varepsilon>\varepsilon_{n}$ then, in the $\varepsilon$-vicinity of $k_{\mu}$, the minimum of the maxima of the number of points in the $\omega$-limit distribution support does not exceed $n$.

In order to understand this estimate it is sufficient to consider system (1.1) with $k_{\mu}$ of the form (2.11). In this case for any dependence $\mu(t)$ the averages (2.9) have the form

$$
\left\langle k_{\mu(t)}\right\rangle_{t}=\frac{1}{t} \int_{0}^{t} k_{\mu(\tau)} \mathrm{d} \tau=\varphi_{0}(x)+\sum_{i=1}^{n} \varphi_{i}(x) a_{i} .
$$

where $a_{i}$ are some numbers. The ensemble of the functions (2.12) for various $a_{i}$ forms a $n$-dimensional linear manifold. How many points of the global maximum (equal to zero) could a function of this family have? 
Generally speaking, it can have any number of maxima. However, it seems obvious, that "usually" one function has only one point of global maximum, while it is "improbable" that the maximum value is zero. At least, with an arbitrary small perturbation of the given function, we can achieve for the point of the global maximum to be unique and the maximum value be non-zero.

In a one-parametric family of functions there may occur zero value of the global maximum, which cannot be eliminated by a small perturbation, and individual functions of the family may have two global maxima.

In the general case we can state, that "usually" each function of the $n$-parametric family (2.12) can have not more than $n$ points of the zero global maximum (of course, there may be less, and the global maximum is, as a rule, not equal to zero at all for the majority of functions of the family). What "usually" means here requires a special explanation given in the next section.

In application $k_{\mu}$ is often represented by an integral operator, linear or nonlinear. In this case the form (2.11) corresponds to the kernels of integral operators, represented in a form of the sums of functions' products. For example, the reproduction coefficient of the following form

$$
\begin{gathered}
k_{\mu}=\varphi_{0}(x)+\int K(x, y) \mu(y) \mathrm{d} y, \\
\text { where } K(x, y)=\sum_{i=1}^{n} \varphi_{i}(x) g_{i}(y),
\end{gathered}
$$

has also the form $(2.11)$ with $\psi_{i}(\mu)=\int g_{i}(y) \mu(y) \mathrm{d} y$.

The linear reproduction coefficients occur in applications rather frequently. For them the problem of the minimax estimation of the number of points in the $\omega$-limit distribution support is reduced to the question of the accuracy of approximation of the linear integral operator by the sums of kernels-products (2.13).

\subsection{Almost Finite Sets and "Almost Always"}

In this section, some auxiliary propositions and definitions are presented. The supports of the $\omega$-limit distributions for the systems with inheritance were characterized by the optimality principle. These supports consist of points of global maximum of the average reproduction coefficient. We can a priori (without studying the solutions in details) characterize the compact set that includes all possible average reproduction coefficients. Hence, we get a problem: how to describe the set of global maximum for all functions from generic compact set of functions. First of all, any closed subset $M \subset X$ is a set of global maximum of a continuous function, for example, of the function $f(x)=-\rho(x, M)$, where $\rho(x, M)$ is the distance between a set and a point: $\rho(x, M)=\inf _{y \in M} \rho(x, y)$, and $\rho(x, y)$ is the distance between points. Nevertheless, we can expect that one generic function has one point of global maximum, in a generic one-parametric family might exist functions with two points of global maximum, etc. How these expectations meet the exact results? What does the 
notion "generic" mean? What can we say about sets of global maximum of functions from a generic compact family? In this section we answer these questions.

"Almost always", "typically", "generically" a function has only one point of global maximum. This sentence should be given an rigorous meaning. Formally it is not difficult, but haste is dangerous when defining "genericity".

Here are some examples of correct but useless statements about "generic" properties of function: Almost every continuous function is not differentiable; Almost every $C^{1}$-function is not convex. Their meaning for applications is most probably this: the genericity used above for continuous functions or for $C^{1}$-function is irrelevant to the subject.

Most frequently the motivation for definitions of genericity is found in such a situation: given $n$ equations with $m$ unknowns, what can we say about the solutions? The answer is: in a typical situation, if there are more equations, than the unknowns $(n>m)$, there are no solutions at all, but if $n \leq m$ ( $\mathrm{n}$ is less or equal to $\mathrm{m}$ ), then, either there is a $(m-n)$-parametric family of solutions, or there are no solutions.

The best known example of using this reasoning is the Gibbs phase rule in classical chemical thermodynamics. It limits the number of co-existing phases. There exists a wellknown example of such reasoning in mathematical biophysics too. Let us consider a medium where $n$ species coexist. The medium is assumed to be described by $m$ parameters $s_{j}$. Dynamics of these parameters depends on the organisms. In the simplest case, the medium is a well-mixed solution of $m$ substances. Let the organisms interact through the medium, changing its parameters - concentrations of $m$ substances. It can be formalized by a system of equation:

$$
\begin{aligned}
& \frac{\mathrm{d} \mu_{i}}{\mathrm{~d} t}=k_{i}\left(s_{1}, \ldots, s_{m}\right) \times \mu_{i}(i=1, \ldots n) ; \\
& \frac{\mathrm{d} s_{j}}{\mathrm{~d} t}=q_{j}\left(s_{1}, \ldots, s_{m}, \mu_{1}, \ldots, \mu_{n}\right)(j=1, \ldots m),
\end{aligned}
$$

In a steady state, for each of the coexisting species we have an equation with respect to the state of the medium: the corresponding reproduction coefficient $k_{i}$ is zero. So, the number of such species cannot exceed the number of parameters of the medium. In a typical situation, in the $m$-parametric medium in a steady state there can exist not more than $m$ species. This is the concurrent exclusion principle in the G. Gause form [26]. Here, the main hypothesis about interaction of organisms with the media is that the number of essential components of the media is bounded from above by $m$ and increase of the number of species does not extend the list of components further. Dynamics of parameters depends on the organisms, but their nomenclature is fixed.

This concurrent exclusion principle allows numerous generalizations [56, 57, 53, 17, 59]. Theorem of the natural selection efficiency may be also considered as its generalization.

Analogous assertion for a non-steady state coexistence of species in the case of equations (2.14) is not true. It is not difficult to give an example of stable coexistence under oscillating conditions of $n$ species in the $m$-parametric medium at $n>m$.

But, if $k_{i}\left(s_{1}, \ldots, s_{m}\right)$ are linear functions of $s_{1}, \ldots, s_{m}$, then for non-stable conditions we have the concurrent exclusion principle, too. In that case, the average in time of the 
reproduction coefficient is the reproduction coefficient for the average state of the medium:

$$
\left\langle k_{i}\left(s_{1}(t), \ldots, s_{m}(t)\right)\right\rangle=k_{i}\left(\left\langle s_{1}\right\rangle, \ldots,\left\langle s_{m}\right\rangle\right)
$$

because of linearity. If $\left\langle x_{i}\right\rangle \neq 0$ then $k_{i}\left(\left\langle s_{1}\right\rangle, \ldots,\left\langle s_{m}\right\rangle\right)=0$, and we obtain the non-stationary concurrent exclusion principle "in average". And again, it is valid "almost always".

The non-stationary concurrent exclusion principle "in average" is valid for linear reproduction coefficients. This is a combination of the Volterra [83] averaging principle and the Gause principle,

It is worth to mention that, for our basic system (1.1), if $k_{\mu}$ are linear functions of $\mu$, then the average in time of the reproduction coefficient $k_{\mu(t)}$ is the reproduction coefficient for the average $\mu(t)$ because of linearity. Therefore, the optimality principle (2.10) for the average reproduction coefficient $k^{*}$, transforms into the following optimality principle for the reproduction coefficient $k_{\langle\mu\rangle}$ of the average distribution $\langle\mu\rangle$

$$
\begin{aligned}
& k_{\langle\mu\rangle}(x)=0 \text { if } x \in \operatorname{supp} \mu^{*} \\
& k_{\langle\mu\rangle}(x) \leq 0 \text { if } x \in \operatorname{supp} \mu(0) .
\end{aligned}
$$

(the generalized Volterra averaging principle [83]).

Formally, various definitions of genericity are constructed as follows. All systems (or cases, or situations and so on) under consideration are somehow parameterized - by sets of vectors, functions, matrices etc. Thus, the "space of systems" $Q$ can be described. Then the "thin sets" are introduced into $Q$, i.e. the sets, which we shall later neglect. The union of a finite or countable number of thin sets, as well as the intersection of any number of them should be thin again, while the whole $Q$ is not thin. There are two traditional ways to determine thinness.

1. A set is considered thin when it has measure zero. This is resonable for a finitedimensional case, when there is the standard Lebesgue measure - the length, the area, the volume.

2. But most frequently we deal with the functional parameters. In that case it is common to restore to the second definition, according to which the sets of Baire first category are negligible. The construction begins with nowhere dense sets. The set $Y$ is nowhere dense in $Q$, if in any nonempty open set $V \subset Q$ (for example, in a ball) there exists a nonempty open subset $W \subset V$ (for example, a ball), which does not intersect with $Y$ : $W \cap Y=\emptyset$. Roughly speaking, Y is "full of holes" - in any neighborhood of any point of the set $Y$ there is an open hole. Countable union of nowhere dense sets is called the set of first category. The second usual way is to define thin sets as the sets of first category. A residual set (a "thick" set) is the complement of a set of the first category.

For the second approach, the Baire category theorem is important: In a non-empty complete metric space, any countable intersection of dense, open subsets is non-empty. 
But even the real line $R$ can be divided into two sets, one of which has zero measure, the other is of first category. The genericity in the sense of measure and the genericity in the sense of category considerably differ in the applications where both of these concepts can be used. The conflict between the two main views on genericity stimulated efforts to invent new and stronger approaches.

Systems (1.1) were parameterized by continuous maps $\mu \mapsto k_{\mu}$. Denote by $Q$ the space of these maps $M \rightarrow C(X)$ with the topology of uniform convergence on $M$. It is a Banach space. Therefore, we shall consider below thin sets in a Banach space $Q$. First of all, let us consider $n$-dimensional affine compact subsets of $Q$ as a Banach space of affine maps $\Psi:[0,1]^{n} \rightarrow Q\left(\Psi\left(\alpha_{1}, \ldots \alpha_{n}\right)=\sum_{i} \alpha_{i} f_{i}+\varphi, \alpha_{i} \in[0,1], f_{i}, \varphi \in Q\right)$ in the maximum norm. For the image of a map $\Psi$ we use the standard notation $\operatorname{im} \Psi$.

Definition 2.1. A set $Y \subset Q$ is $n$-thin, if the set of affine maps $\Psi:[0,1]^{n} \rightarrow Q$ with non-empty intersection $\operatorname{im} \Psi \cap Y \neq \emptyset$ is the set of first category.

All compact sets in infinite-dimensional spaces and closed linear subspaces with codimension greater then $n$ are $n$-thin. If $\operatorname{dim} Q \leq n$, then only empty set is $n$-thin in $Q$. The union of a finite or countable number of $n$-thin sets, as well as the intersection of any number of them is $n$-thin, while the whole $Q$ is not $n$-thin.

Let us consider compact subsets in $Q$ parametrized by points of a compact space $K$. It can be presented as a Banach space $C(K, Q)$ of continuous maps $K \rightarrow Q$ in the maximum norm.

Definition 2.2. A set $Y \subset Q$ is completely thin, if for any compact $K$ the set of continuous maps $\Psi: K \rightarrow Q$ with non-empty intersection $\operatorname{im} \Psi \cap Y \neq \emptyset$ is the set of first category.

A set $Y$ in the Banach space $Q$ is completely thin, if for any compact set $K$ in $Q$ and arbitrary positive $\varepsilon>0$ there exists a vector $q \in Q$, such that $\|q\|<\varepsilon$ and $K+q$ does not intersect $Y:(K+q) \cap Y=\emptyset$. All compact sets in infinite-dimensional spaces and closed linear subspaces with infinite codimension are completely thin. Only empty set is completely thin in a finite-dimensional space. The union of a finite or countable number of completely thin sets, as well as the intersection of any number of them is completely thin, while the whole $Q$ is not completely thin.

Proposition 2.1. If a set $Y$ in the Banach space $Q$ is completely thin, then for any compact metric space $K$ the set of continuous maps $\Psi: K \rightarrow Q$ with non-empty intersection $\operatorname{im} \Psi \cap$ $Y \neq \emptyset$ is completely thin in the Banach space $C(K, Q)$.

Proof. It is sufficient to mention that for any compact $P$ the space $C(P, C(K, Q))$ of continuous maps $P \rightarrow C(K, Q)$ is isomorphic to the space $C(P \times K, Q)$, and $P \times K$ is compact again.

Below the wording "almost always" means: the set of exclusions is completely thin. The main result presented in this section sounds as follows: almost always the sets of global maxima of functions from a compact set are uniformly almost finite.

Proposition 2.2. Let $X$ have no isolated points. Then almost always a function $f \in C(X)$ 
has nowhere dense set of zeros $\{x \in X \mid f(x)=0\}$ (the set of exclusions is completely thin in $C(X))$.

Proof. Let us mention that the topology of $X$ has a countable base $\left\{U_{i}\right\}_{i=1}^{\infty}$. For any $i$ the set of functions

$$
\text { Annul } U_{i}=\left\{f \in C(X) \mid f(x)=0 \text { for all } x \in U_{i}\right\}
$$

is closed subspace of $C(X)$ (even an ideal) with infinite codimension. For any compact set $K \subset C(X)$ the sets of shift vectors

$$
V_{i}=\left\{y \in C(X) \mid(K+y) \cap \operatorname{Annul} U_{i}=\emptyset\right\}
$$

is open and dense in $C(X)$. Hence, the set of shifts $\cap_{i} V_{i}$ is dense residual set in $C(X)$.

After combination Proposition 2.2 with Proposition 2.1 we get the following

Proposition 2.3. Let $X$ have no isolated points. Then for any compact space $K$ and almost every continuous map $\Psi: K \rightarrow C(X)$ all functions $f \in \operatorname{im} \Psi$ have nowhere dense sets of zeros (the set of exclusions is completely thin in $C(K, C(X))$ ).

In other words, in almost every compact family of continuous functions all the functions have nowhere dense sets of zeros.

In construction of "almost finite" sets we follow a rather old idea that was used by Liouville in construction of his "almost rational" transcendental numbers [12]. A Liouville number is a transcendental number which has very close rational number approximations. An irrational number $\beta$ is called a Liouville number if, for each $n$, there exist integers $p$ and $q$ such that

$$
\left|\beta-\frac{p}{q}\right|<\frac{1}{q^{n}}
$$

An example of such a number gives Liouville's constant, sometimes also called Liouville's number, that is the real number defined by

$$
L=\sum_{n=1}^{\infty} 10^{-n !} .
$$

Liouville's constant is a decimal fraction with a 1 in each decimal place corresponding to a factorial, and zeros everywhere else. It was the first decimal constant to be proven transcendental.

In some sense, almost all real numbers are the Liouville numbers: the set of all Liouville numbers is the residual set. To prove this statement let us enumerate all the rational numbers: $r_{1}, r_{2}, \ldots, r_{n}=p_{n} / q_{n}$. The following set $U_{\epsilon}$ is open and dense on the real line:

$$
U_{\epsilon}=\bigcup_{n=1}^{\infty}\left\{\beta|| \beta-\frac{p_{n}}{q_{n}} \mid<\frac{\epsilon}{q_{n}^{n}}\right\} .
$$


The intersection of $U_{\epsilon}$ for $\epsilon=1 / 2,1 / 4,1 / 8, \ldots$

$$
U=\bigcap_{k=1}^{\infty} U_{\frac{1}{2^{k}}}
$$

is the residual set, and numbers from $U$ are the Liouville numbers. On the other hand, $U$ has zero Lebesgue measure, and it gives us an example of dividing the real line on the set of first category (the complement of $U$ ) and the set of the zero measure $U$.

Let us consider a space of closed subsets of the compact metric space $X$ endowed by the Hausdorff distance. The Hausdorff distance between closed subsets of $X$ is

$$
\operatorname{dist}(A, B)=\max \left\{\sup _{x \in A} \inf _{x \in B} \rho(x, y), \sup _{x \in B} \inf _{x \in A} \rho(x, y)\right\} .
$$

The almost finite sets were introduced in [29] for description of the typical sets of maxima for continuous functions from a compact set. This definition depends on an arbitrary sequence $\varepsilon_{n}>0, \varepsilon_{n} \rightarrow 0$. For any such sequence we construct a class of subsets $Y \subset X$ that can be approximated by finite set faster than $\varepsilon_{n} \rightarrow 0$, and for families of sets we introduce a notion of uniform approximation by finite sets faster than $\varepsilon_{n} \rightarrow 0$ :

Definition 2.3. Let $\varepsilon_{n}>0, \varepsilon_{n} \rightarrow 0$. The set $Y \subset X$ can be approximated by finite sets faster than $\varepsilon_{n} \rightarrow 0\left(\varepsilon_{n}>0\right)$, if for any $\delta>0$ there exists a finite set $S_{N}$ such that $\operatorname{dist}\left(S_{N}, Y\right)<\delta \varepsilon_{N}$. The sets of family $\mathbb{Y}$ can be uniformly approximated by finite sets faster than $\varepsilon_{n} \rightarrow 0$, if for any $\delta>0$ there exists such a number $N$ that for any $Y \in \mathbb{Y}$ there exists a finite set $S_{N}$ such that $\operatorname{dist}\left(S_{N}, Y\right)<\delta \varepsilon_{N}$.

The simplest example of almost finite set on the real line for a given $\varepsilon_{n} \rightarrow 0\left(\varepsilon_{n}>0\right)$ is the sequence $\varepsilon_{n} / n$. If $\varepsilon_{n}<$ const $/ n$, then the set $Y$ on the real line which can be approximated by finite sets faster than $\varepsilon_{n} \rightarrow 0$ have zero Lebesgue measure. At the same time, it is nowhere dense, because it can be covered by a finite number of intervals with an arbitrary small sum of lengths (hence, in any interval we can find a subinterval free of points of $Y$ ).

Let us study the sets of global maxima argmax $f$ for continuous functions $f \in C(X)$. For each $f \in C(X)$ and any $\epsilon>0$ there exists $\phi \in C(X)$ such that $\|f-\phi\| \leq \epsilon$ and $\operatorname{argmax} \phi$ consists of one point. Such a function $\phi$ can be chosen in the form

$$
\phi(x)=f(x)+\frac{\epsilon}{1+\rho\left(x, x_{0}\right)^{2}},
$$

where $x_{0}$ is an arbitrary element of $\operatorname{argmax} f$. In this case $\operatorname{argmax} \phi=\left\{x_{0}\right\}$.

Hence, the set $\operatorname{argmax} f$ can be reduced to one point by an arbitrary small perturbations of the function $f$. On the other hand, it is impossible to extend significantly the set $\operatorname{argmax} f$ by a sufficiently small perturbation, the dependence of this set on $f$ is semicontinuous in the following sense.

Proposition 2.4. For given $f \in C(X)$ and any $\varepsilon>0$ there exists $\delta>0$ such that, whenever $\|f-\phi\|<\delta$, then

$$
\max _{x \in \operatorname{argmax} \phi} \min _{y \in \operatorname{argmax} f} \rho(x, y)<\varepsilon
$$


Proof. In order to derive the dependence of $\delta$ on $\varepsilon$ in Proposition 2.4, we can use the following auxiliary function:

$$
\eta_{f}(r)=\min _{\rho(x, \operatorname{argmax} f) \geq r}\left\{\max _{X} f-f(x)\right\}
$$

where $\rho(x, \operatorname{argmax} f)$ is the distance between a set and a point.

The function $\eta_{f}(r)$ is monotone nondecreasing, $\eta_{f}(0)=0$, and $\eta_{f}(r)>0$ for $r>0$. We can take in Proposition 2.4 any $\delta<\eta_{f}(\varepsilon)$, for example, $\delta=\eta_{f}(\varepsilon) / 2$.

In particular, if the set $\operatorname{argmax} f$ consists of one point $x_{0}$, then for sufficiently small perturbations of $f$ the set argmax remains in an arbitrary small ball near $x_{0}$.

These constructions can be generalized onto $n$-parametric affine compact families of continuous functions. Let us consider affine maps of the cube $[0,1]^{k}$ into $C(X), \Phi:[0,1]^{k} \rightarrow$ $C(X)$. The space of all such maps is a Banach space endowed with the maximum norm.

Proposition 2.5. For any affine map $\Phi:[0,1]^{k} \rightarrow C(X)$ and an arbitrary $\epsilon>0$ there exists such a continuous function $\psi \in C(X)$, that $\|\psi\|<\epsilon$ and the set $\operatorname{argmax}(f+\psi)$ includes not more than $k+1$ points for all $f \in \operatorname{im} \Phi$.

Proof. To prove this Proposition 2.5 we can use the following Lemma.

Lemma 2.1. Let $Q \subset C(X)$ be a compact set of functions, $\varepsilon>0$. Then there are a finite set $Y \subset X$ and a function $\phi \in C(X)$ such that $\|\phi\|<\varepsilon$, and any function $f \in Q+\phi$ achieves its maximum only on $Y: \operatorname{argmax} f \subset Y$.

To find the shift function $\phi$ let us use the following auxiliary functions: for $f \in C(X)$

$$
\gamma_{f}(r)=\max _{\rho(x, y) \leq r}|f(x)-f(y)|
$$

The function $\gamma_{f}(r)$ is monotone nondecreasing, $\gamma_{f}(0)=0$, and $\gamma_{f}(r) \rightarrow 0$ for $r \rightarrow 0$. Sometimes one calls it the uniform continuity module of $f$. For the compact of functions $Q \subset C(X)$ we can also define the uniform continuity module:

$$
\gamma_{Q}(r)=\max _{f \in Q} \gamma_{f}(r)=\max _{f \in Q, \rho(x, y) \leq r}|f(x)-f(y)|
$$

The function $\gamma_{Q}(r)$ is monotone nondecreasing, $\gamma_{Q}(0)=0$, and $\gamma_{Q}(r) \rightarrow 0$ for $r \rightarrow 0$. For general compact space $X$ the function $\gamma_{f}(r), \gamma_{Q}(r)$ might be not continuous at points $r \neq 0$. Instead of them we can use their continuous majorants, for example, the concave closures of these functions. Hypograph of a function $\gamma(r)$, denoted hyp $(\gamma)$, is the set $\{(r, g) \mid g \leq \gamma(r)\}$. Note that the hypograph is the region below the graph of $\gamma$. The concave closure of $\gamma(r)$ (denoted as $\overline{\operatorname{conc}} \gamma$ ) is the function that has as hypograph the closure of the convex hull of $\operatorname{hyp}(\gamma)$ (that is, the smallest closed and convex set containing hyp $(\gamma)$ ). If a function $\gamma(r)$ on an interval $[0, R]$ is monotone nondecreasing, bounded, $\gamma(0)=0$, and $\gamma(r) \rightarrow 0$ for $r \rightarrow 0$ (that is $\gamma(r)$ is continuous at the point $r=0$ ), then the function $\overline{\operatorname{conc}} \gamma$ on the interval $[0, R]$ is 
continuous, monotone nondecreasing, $\overline{\operatorname{conc}} \gamma(r) \geq \gamma(r)$ for all $r \in[0, R], \overline{\mathrm{conc}} \gamma(0)=\gamma(0)=0$ and $\overline{\operatorname{conc}} \gamma(R)=\gamma(R)$.

To exclude trivial case, let us assume that there is a non-constant function in $Q$. Then $\max \gamma_{Q}(r)>0$, and for given $\gamma>0, \gamma<\max \gamma_{Q}(r)$ we can find $r(\gamma)$, a unique solution of the equation $\overline{\operatorname{conc}} \gamma_{Q}(r)=\gamma$. This $r(\gamma)$ is continuous function for $0 \leq \gamma<\max \gamma_{Q}(r)$.

For the given $\varepsilon>0$, let us find in $X$ a finite $r(\varepsilon / 2)$-net $\left\{x_{1}, x_{2}, \ldots, x_{N}\right\} \subset X$. For each $x_{i}$ we define a $\varepsilon$-small "cap function"

$$
\begin{aligned}
& \phi_{i}(x)=\frac{4}{3}\left(\frac{\varepsilon}{2}-\gamma_{Q}\left(\rho\left(x, x_{i}\right) \frac{r_{1}}{r_{2}}\right)\right) \text { if } \rho\left(x, x_{i}\right)<r_{2}, \\
& \phi_{i}(x)=0 \text { if } \rho\left(x, x_{i}\right) \geq r_{2},
\end{aligned}
$$

where $r_{1}=r(\varepsilon / 2), r_{2}=\frac{1}{2} \min \left\{r_{1}, \frac{1}{2} \min _{i \neq j} \rho\left(x_{i}, x_{j}\right)\right\}$.

If $\rho\left(x, x_{i}\right) \leq \varepsilon / 2$ and $f \in Q$, then $\left|f(x)-f\left(x_{i}\right)\right|<\phi_{i}(x)$. If $\phi_{i}(x) \neq 0$ then $\phi_{j}(x)=0$ for all $j \neq i ;\left\|\phi_{i}(x)\right\|<\varepsilon$ and $\phi_{i}(x) \geq 0$ for each $i$.

We can define the shift function $\phi$ in Lemma 2.1 as

$$
\phi(x)=\sum_{i=1}^{N} \phi_{i}(x) .
$$

Lemma 2.1 allows us to reduce some problems concerning global maxima of continuous functions from a compact sets $Q \subset C(X)$ to questions about functions on finite subsets in $X$. In particular, Proposition 2.5 reduces to a question about existence of non-trivial solutions for finite systems of linear equations. Let us consider functions on the finite net $\left\{x_{1}, x_{2}, \ldots, x_{N}\right\} \subset X$. For each affine map $\Phi:[0,1]^{k} \rightarrow C(X)\left(\Phi\left(\alpha_{1}, \ldots \alpha_{n}\right)=\sum_{i} \alpha_{i} f_{i}+\varphi\right.$, $\left.\alpha_{i} \in[0,1], f_{i}, \varphi \in C(X)\right)$ the values $\Phi\left(\alpha_{1}, \ldots \alpha_{n}\right)\left(x_{i}\right)$ are linear (non-homogeneous) functions on the $k$-cube $[0,1]^{k}$. For any $q$ different points $x_{i_{1}}, \ldots x_{i_{q}}$ of the net the system of equations

$$
\Phi\left(\alpha_{1}, \ldots \alpha_{n}\right)\left(x_{i_{1}}\right)=\ldots=\Phi\left(\alpha_{1}, \ldots \alpha_{n}\right)\left(x_{i_{q}}\right)
$$

can, generically, have a solution in the $k$-cube $[0,1]^{k}$ only for $q \leq k+1$. The degenerated case with solutions for $q>k+1$ can be destroyed by an arbitrary small perturbation. This simple remark together with Lemma 2.1 proves Proposition 2.5.

Note, that Proposition 2.5 and Lemma 2.1 demonstrate us different sources of discreteness: in Lemma 2.1 it is the approximation of a compact set by a finite net, and in Proposition 2.5 it is the connection between the number of parameters and the possible number of global maximums in a $k$-parametric family of functions. There is no direct connection between $N$ and $k$ values, and it might be that $N \gg k$. For smooth functions in finitedimensional real space polynomial approximations can be used instead of Lemma 2.1 in order to prove the analogue of Proposition 2.5.

For any compact $K$ the space of continuous maps $C(K, C(X))$ is isomorphic to the space of continuous functions $C(K \times X)$. Each continuous map $F: K \rightarrow C(X)$ can be 
approximated with an arbitrary accuracy $\varepsilon>0$ by finite sums of the following form $(k \geq 0)$ :

$$
\begin{aligned}
& F(y)(x)=\sum_{i=1}^{k} \alpha_{i}(y) f_{i}(x)+\varphi(x)+o, \\
& y \in K, x \in X, 0 \leq \alpha_{i} \leq 1, f_{i}, \varphi \in C(X),|o|<\varepsilon .
\end{aligned}
$$

Each set $f_{i}, \varphi \in C(X)$ generates a map $\Phi:[0,1]^{k} \rightarrow C(X)$. A dense subset in the space of these maps satisfy the statement of Proposition 2.5: each function from im $\Phi$ has not more than $k+1$ points of global maximum. Let us use for this set of maps $\Phi$ notation $\mathbf{P}_{k}$, for the correspondent set of the maps $F: K \rightarrow C(X)$, which have the form of finite sums (2.16), notation $\mathbf{P}_{k}^{K}$, and $\mathbf{P}^{K}=\cup_{k} \mathbf{P}_{k}^{K}$.

For each $\Phi \in \mathbf{P}_{k}^{K}$ and any $\varepsilon>0$ there is $\delta=\delta_{\Phi}(\varepsilon)>0$ such that, whenever $\|\Psi-\Phi\|<$ $\delta_{\Phi}(\varepsilon)$, the set $\operatorname{argmax} f$ belongs to a union of $k+1$ balls of radius $\varepsilon$ for any $f \in \operatorname{im} \Psi$ (Proposition 2.4).

Let us introduce some notations: for $k \geq 0$ and $\varepsilon>0$

$$
\mathbf{U}_{k, \varepsilon}^{K}=\left\{\Psi \in C(K, C(X))|| \Psi-\Phi \|<\delta_{\Phi}(\varepsilon) \text { for some } \Phi \in \mathbf{P}_{k}^{K}\right\} ;
$$

for $\varepsilon_{i}>0, \varepsilon_{i} \rightarrow 0$

$$
\mathbf{V}_{\left\{\varepsilon_{i}\right\}}^{K}=\bigcup_{k=0}^{\infty} \mathbf{U}_{k, \varepsilon_{k}}^{K}
$$

and, finally,

$$
\mathbf{W}_{\left\{\varepsilon_{i}\right\}}^{K}=\bigcap_{s=1}^{\infty} \mathbf{V}_{\left\{\frac{1}{2^{s}} \varepsilon_{i}\right\}}^{K} .
$$

The set $\mathbf{P}^{K}$ is dense in $C(K, C(X))$. Any $F \in \mathbf{P}^{K}$ has the form of finite sum (2.16), and any $f \in \operatorname{im} F$ has not more than $k+1$ point of global maximum, where $k$ is the number of summands in presentation (2.16). The sets $\mathbf{V}_{\left\{\varepsilon_{i}\right\}}^{K}$ are open and dense in the Banach space $C(K, C(X))$ for any sequence $\varepsilon_{i}>0, \varepsilon_{i} \rightarrow 0$. The set $\mathbf{W}_{\left\{\varepsilon_{i}\right\}}^{K}$ is intersection of countable number of open dense sets. For any $F \in \mathbf{W}_{\left\{\varepsilon_{i}\right\}}^{K}$ the sets of the family $\{\operatorname{argmax} f \mid f \in \operatorname{im} F\}$ can be uniformly approximated by finite sets faster than $\varepsilon_{n} \rightarrow 0$. It is proven that this property is typical in the Banach space $C(K, C(X))$ in the sense of category. In order to prove that the set of exclusions is completely thin in $C(K, C(X))$ it is sufficient to use the approach of Proposition 2.1. Note that for arbitrary compact space $Q$ the set of continuous maps $Q \rightarrow C(K, C(X))$ in the maximum norm is isomorphic to the spaces $C(Q \times K, C(X))$ and $C(Q \times K \times X)$. The space $Q \times K$ is compact. We can apply the previous construction to the space $C(Q \times K, C(X))$ for arbitrary compact $Q$ and get the result: the set of exclusion is completely thin in $C(K, C(X))$.

In the definition of $\mathbf{W}_{\left\{\varepsilon_{i}\right\}}^{K}$ we use only one sequence $\varepsilon_{i}>0, \varepsilon_{i} \rightarrow 0$. Of course, for any finite or countable set of sequences the intersection of correspondent sets $\mathbf{W}_{\left\{\varepsilon_{i}\right\}}^{K}$ is also a 
residual set, and we can claim that almost always the sets of $\{\operatorname{argmax} f \mid f \in \operatorname{im} F\}$ can be uniformly approximated by finite sets faster than $\varepsilon_{n} \rightarrow 0$ for all given sequences. Let us mention that the set of all recursive enumerable countable sets is also countable and not continuum. This observation is very important for algorithmic foundations of probability theory [54]. Let $\boldsymbol{L}$ be a set of all sequences of real numbers $\varepsilon_{n}>0, \varepsilon_{n} \rightarrow 0$ with the property: for each $\left\{\varepsilon_{n}\right\} \in \boldsymbol{L}$ the rational hypograph $\left\{(n, r) \mid \varepsilon_{n}>r \in \boldsymbol{Q}\right\}$ (where $\boldsymbol{Q}$ is the set of rational numbers) is recursively enumerable. The set

$$
\mathbf{W}^{K}=\bigcap_{\left\{\varepsilon_{i}\right\} \in \boldsymbol{L}} \mathbf{W}_{\left\{\varepsilon_{i}\right\}}^{K}
$$

is a residual set again and almost always the sets of $\{\operatorname{argmax} f \mid f \in \operatorname{im} F\}$ can be uniformly approximated by finite sets faster than $\varepsilon_{n} \rightarrow 0$ for all given sequences from $\boldsymbol{L}$.

\subsection{Selection Efficiency}

The first application of the extremal principle for the $\omega$-limit sets is the theorem of the selection efficiency. The dynamics of a system with inheritance leads indeed to a selection in the limit $t \rightarrow \infty$. In the typical situation, a diversity in the limit $t \rightarrow \infty$ becomes less than the initial diversity. There is an efficient selection for the "best". The basic effects of selection are formulated below. Let $X$ be compact metric space without isolated points.

\section{Theorem 2.1. (Theorem of selection efficiency.)}

1. For almost every system (1.1) the support of any $\omega$-limit distribution is nowhere dense in $X$ (and it has the Lebesgue measure zero for Euclidean space).

2. Let $\varepsilon_{n}>0, \varepsilon_{n} \rightarrow 0$ be an arbitrary chosen sequence. The following statement is true for almost every system (1.1). Let the support of the initial distribution be the whole $X$. Then the support of any $\omega$-limit distribution can be approximated by finite sets uniformly faster than $\varepsilon_{n} \rightarrow 0$.

The set of exclusive systems that do not satisfy the statement 1 or 2 is completely thin.

Remark. These properties hold for the continuous reproduction coefficients. It is wellknown, that it is dangerous to rely on the genericity among continuous functions. For example, almost all continuous functions are nowhere differentiable. But the properties 1, 2 hold also for the smooth reproduction coefficients on the manifolds and sometimes allow to replace the "almost finiteness" by simply finiteness.

Proof. To prove the first statement, it is sufficient to refer to Proposition 2.3. In order to clarify the second part of this theorem, note that:

1. Support of an arbitrary $\omega$-limit distribution $\mu^{*}$ consist of points of global maximum of the average reproduction coefficient on a support of the initial distribution. The corresponding maximum value is zero. 
2. Almost always a function has only one point of global maximum, and corresponding maximum value is not 0 .

3. In a one-parametric family of functions almost always there may occur zero values of the global maximum (at one point), which cannot be eliminated by a small perturbation, and individual functions of the family may stably have two global maximum points.

4. For a generic $n$-parameter family of functions, there may exist stably a function with $n$ points of global maximum and with zero value of this maximum.

5. Our phase space $M$ is compact. The set of corresponding reproduction coefficients $k_{M}$ in $C(X)$ for the given map $\mu \rightarrow k_{\mu}$ is compact too. The average reproduction coefficients belong to the closed convex hull of this set $\overline{\operatorname{conv}}\left(k_{M}\right)$. And it is compact too.

6. A compact set in a Banach space can be approximated by compacts from finitedimensional linear manifolds. Generically, in a space of continuous functions, a function, which belongs such a $n$-dimensional compact, can have not more than $n$ points of global maximum with zero maximal value.

The rest of the of proof of the second statement is purely technical. Some technical details are presented in the previous section. The easiest demonstration of the "natural" character of these properties is the demonstration of instability of exclusions: If, for example, a function has several points of global maxima, then with an arbitrary small perturbation (for all usually used norms) it can be transformed into a function with the unique point of global maximum. However "stable" does not always mean "dense". The discussed properties of the system (1.1) are valid in a very strong sense: the set of exclusion is completely thin.

\subsection{Gromov's Interpretation of Selection Theorems}

In his talk [38], M. Gromov offered a geometric interpretation of the selection theorems. Let us consider dynamical systems in the standard $m$-simplex $\sigma_{m}$ in $m+1$-dimensional space $R^{m+1}$ :

$$
\sigma_{m}=\left\{x \in R^{m+1} \mid x_{i} \geq 0, \sum_{i=1}^{m+1} x_{i}=1\right\} .
$$

We assume that simplex $\sigma_{m}$ is positively invariant with respect to these dynamical systems: if the motion starts in $\sigma_{m}$ at some time $t_{0}$, then it remains in $\sigma_{m}$ for $t>t_{0}$. Let us consider the motions that start in the simplex $\sigma_{m}$ at $t=0$ and are defined for $t>0$.

For large $m$, almost all volume of the simplex $\sigma_{m}$ is concentrated in a small neighborhood of the center of $\sigma_{m}$, near the point $c=\left(\frac{1}{m}, \frac{1}{m}, \ldots, \frac{1}{m}\right)$. Hence, one can expect that a typical motion of a general dynamical system in $\sigma_{m}$ for sufficiently large $m$ spends almost all the time in a small neighborhood of $c$. 
Indeed, the $m$-dimensional volume of $\sigma_{m}$ is $V_{m}=\frac{1}{m !}$. The part of $\sigma_{m}$, where $x_{i} \geq \varepsilon$, has the volume $(1-\varepsilon)^{m} V_{m}$. Hence, the part of $\sigma_{m}$, where $x_{i}<\varepsilon$ for all $i=1, \ldots, m+1$, has the volume $V_{\varepsilon}>\left(1-(m+1)(1-\varepsilon)^{m}\right) V_{m}$. Note, that $(m+1)(1-\varepsilon)^{m} \sim m \exp (-\varepsilon m) \rightarrow 0$, if $m \rightarrow \infty(1>\varepsilon>0)$. Therefore, for $m \rightarrow \infty, V_{\varepsilon}=(1-o(1)) V_{m}$. The volume $W_{\rho}$ of the part of $\sigma_{m}$ with Euclidean distance to the center $c$ less than $\rho>0$ can be estimated as follows: $W_{\rho}>V_{\varepsilon}$ for $\varepsilon \sqrt{m+1}=\rho$, hence $W_{\rho}>\left(1-(m+1)(1-\rho / \sqrt{m+1})^{m}\right) V_{m}$. Finally, $(m+1)(1-\rho / \sqrt{m+1})^{m} \sim m \exp (-\rho \sqrt{m})$, and $W_{\rho}=(1-o(1)) V_{m}$ for $m \rightarrow \infty$. Let us mention here the opposite concentration effect for a $m$-dimensional ball $B_{m}$ : for $m \rightarrow \infty$ the most part of its volume is concentrated in an arbitrary small vicinity of its boundary, the sphere. This effect is the essence of the famous equivalence of microcanonical and canonical ensembles in statistical physics (for detailed discussion see [30]).

Let us consider dynamical systems with an additional property ("inheritance"): all the faces of the simplex $\sigma_{m}$ are also positively invariant with respect to the systems with inheritance. It means that if some $x_{i}=0$ initially at the time $t=0$, then $x_{i}=0$ for $t>0$ for all motions in $\sigma_{m}$. The essence of selection theorems is as follows: a typical motion of a typical dynamical system with inheritance spends almost all the time in a small neighborhood of low-dimensional faces, even if it starts near the center of the simplex.

Let us denote by $\partial_{r} \sigma_{m}$ the union of all $r$-dimensional faces of $\sigma_{m}$. Due to the selection theorems, a typical motion of a typical dynamical system with inheritance spends almost all time in a small neighborhood of $\partial_{r} \sigma_{m}$ with $r \ll m$. It should not obligatory reside near just one face from $\partial_{r} \sigma_{m}$, but can travel in neighborhood of different faces from $\partial_{r} \sigma_{m}$ (the drift effect). The minimax estimation of the number of points in $\omega$-limit distributions through the diameters $\varepsilon_{n}>0$ of the set $\overline{\operatorname{conv}}\left(k_{M}\right)$ is the estimation of $r$.

\subsection{Decreasing Measures of Diversity, Lyapunov Functionals, and Burg Entropy}

The distinguished Lyapunov functionals play important role in kinetics. For physical and chemical systems such a functional is, as a rule, the entropy or some of related functionals. The standard examples are the free (or Helmholz) energy and the free enthalpy (or Gibbs energy). It appears that for system (1.1) there exist generically a plenty of Lyapunov functionals. They can be considered as the decreasing measures of diversity. These functionals are very similar to the entropy, but rather to the Burg entropy [11,31], and not to the classic Boltzmann-Gibbs-Shannon entropy.

Generically, we can assume that the convex compact set $\overline{\operatorname{conv}}\left(k_{M}\right)$ does not include zero. The set of exclusion from this rule is completely thin. Then there exists a continuous functional $l$ on $C(X)$ with positive values on $\overline{\operatorname{conv}}\left(k_{M}\right)$. It is a measure on $X$ (by definition). Of course, values of $\rho$ outside $\operatorname{supp} \mu(0)$ do not have any relation to reality, and it is sufficient to discuss only the case $\operatorname{supp} \mu(0)=X$. Any solution of (1.1) can be presented in a form: $\mu(t)=\rho(t) \mu(0)$, where $\rho(t) \in C(X), \rho(t)>0$ at each $t$, and

$$
\frac{\mathrm{d}(\ln \rho(t))}{\mathrm{d} t}=k_{\mu(t)} .
$$


Hence, the "entropy"

$$
S_{l}[\rho(t)]=-[l, \ln \rho(t)]
$$

monotonically decrease:

$$
\frac{\mathrm{d} S_{l}[\rho(t)]}{\mathrm{d} t}=-\left[l, k_{\mu(t)}\right]<0 .
$$

In order to avoid the dependence of an initial distributions $\mu(0)$ we can restrict the initial system (1.1) onto its invariant subspace, the space of measures which have a form $\mu=\rho \mu^{0}$, where $\mu^{0}$ is a given measure with $\operatorname{supp} \mu^{0}=X$, and $\rho \in C(X)$.

The space $L_{\mu^{0}}^{2}(X)$ is the completion of $C(X)$ with respect to the norm

$$
\|f\|=\left[\mu^{0}, f^{2}\right]^{1 / 2}
$$

It is the Hilbert space. For the scalar product we shall use the notation $(,)_{\mu^{0}}$

The compact convex set $\overline{\operatorname{conv}}\left(k_{M}\right)$ is also compact in $L_{\mu^{0}}^{2}(X)$. The set

$$
D_{\mu^{0}}=\left\{\varphi \in L_{\mu^{0}}^{2}(X):(\varphi, f)_{\mu^{0}}>0 \text { for any } f \in \overline{\operatorname{conv}}\left(k_{M}\right)\right\}
$$

is open in $L_{\mu^{0}}^{2}(X)$. Generically, it is nonempty, and, hence, there is a dense set of continuous functions in $D_{\mu^{0}}$. For any function $g \in D_{\mu^{0}}$ we can define the related "entropy"

$$
S[\rho]=-(\ln \rho, g)_{\mu^{0}}=-\left[\mu^{0}, g \ln \rho\right] .
$$

This entropy is the average of the density logarithm with a weight $-g$, the set of allowed weights depends on reproduction coefficient. The entropy (2.19) decrease for each solution of (1.1) that has a form $\mu(t)=\rho(t) \mu^{0}$ with positive initial condition $(\rho(0)$ is strictly positive function).

The introduced entropies decrease monotonically to minus infinity. It is clear, all measures of diversity, including the classical entropy, should decrease in a result of the selection process. The only question was about the monotonicity of this decreasing.

\section{$3 \quad$ Drift and Mutations}

\subsection{Drift Equations}

So far, we talked about the support of an individual $\omega$-limit distribution. For almost all systems it is small. But this does not mean, that the union of these supports is small even for one solution $\mu(t)$. It is possible that a solution is a finite set of narrow peaks getting in time more and more narrow, moving slower and slower, but not tending to fixed positions, rather continuing to move along its trajectory, and the path covered tends to infinity as $t \rightarrow \infty$.

This effect was not discovered for a long time because the slowing down of the peaks was thought as their tendency to fixed positions For the best of our knowledge, the first detailed 
publication of the drift equations and corresponded types of stability appeared in book [29], first examples of coevolution drift on a line were published in the series of papers [70].

There are other difficulties related to the typical properties of continuous functions, which are not typical for the smooth ones. Let us illustrate them for the distributions over a straight line segment. Add to the reproduction coefficients $k_{\mu}$ the sum of small and narrow peaks located on a straight line distant from each other much more than the peak width (although it is $\varepsilon$-small). However small is chosen the peak's height, one can choose their width and frequency on the straight line in such a way that from any initial distribution $\mu_{0}$ whose support is the whole segment, at $t \rightarrow \infty$ we obtain $\omega$-limit distributions, concentrated at the points of maximum of the added peaks.

Such a model perturbation is small in the space of continuous functions. Therefore, it can be put as follows: by small continuous perturbation the limit behavior of system (1.1) can be reduced onto a $\varepsilon$-net for sufficiently small $\varepsilon$. But this can not be done with the small smooth perturbations (with small values of the first and the second derivatives) in the general case. The discreteness of the net, onto which the limit behavior is reduced by small continuous perturbations, differs from the discreteness of the support of the individual $\omega-$ limit distribution. For an individual distribution the number of points is estimated, roughly speaking, by the number of essential parameters (2.11), while for the conjunction of limit supports - by the number of stages in approximation of $k_{\mu}$ by piece-wise constant functions.

Thus, in a typical case the dynamics of systems (1.1) with smooth reproduction coefficients transforms a smooth initial distributions into the ensemble of narrow peaks. The peaks become more narrow, their motion slows down, but not always they tend to fixed positions.

The equations of motion for these peaks can be obtained in the following way [29]. Let $X$ be a domain in the $n$-dimensional real space, and the initial distributions $\mu_{0}$ be assumed to have smooth density. Then, after sufficiently large time $t$, the position of distribution peaks are the points of the average reproduction coefficient maximum $\left\langle k_{\mu}\right\rangle_{t}(2.9)$ to any accuracy set in advance. Let these points of maximum be $x^{\alpha}$, and

$$
q_{i j}^{\alpha}=-\left.t \frac{\partial^{2}\left\langle k_{\mu}\right\rangle_{t}}{\partial x_{i} \partial x_{j}}\right|_{x=x^{\alpha}} .
$$

It is easy to derive the following differential relations

$$
\begin{aligned}
\sum_{j} q_{i j}^{\alpha} \frac{\mathrm{d} x_{j}^{\alpha}}{\mathrm{d} t} & =\left.\frac{\partial k_{\mu(t)}}{\partial x_{i}}\right|_{x=x^{\alpha}} ; \\
\frac{\mathrm{d} q_{i j}^{\alpha}}{\mathrm{d} t} & =-\left.\frac{\partial^{2} k_{\mu(t)}}{\partial x_{i} \partial x_{j}}\right|_{x=x^{\alpha}} .
\end{aligned}
$$

The exponent coefficients $q_{i j}^{\alpha}$ remain time dependent even when the distribution tends to a $\delta$-function. It means (in this case) that peaks became infinitely narrow. Nevertheless, it is possible to change variables and represent the weak* tendency to stationary discrete distribution as usual tendency to a fixed points, see (3.24) below. 
These relations (3.20) do not form a closed system of equations, because the right-hand parts are not functions of $x_{i}^{\alpha}$ and $q_{i j}^{\alpha}$. For sufficiently narrow peaks there should be separation of the relaxation times between the dynamics on the support and the dynamics of the support: the relaxation of peak amplitudes (it can be approximated by the relaxation of the distribution with the finite support, $\left\{x^{\alpha}\right\}$ ) should be significantly faster than the motion of the locations of the peaks, the dynamics of $\left\{x^{\alpha}\right\}$. Let us write the first term of the corresponding asymptotics [29].

For the finite support $\left\{x^{\alpha}\right\}$ the distribution is $\mu=\sum_{\alpha} N_{\alpha} \delta\left(x-x^{\alpha}\right)$. Dynamics of the finite number of variables, $N_{\alpha}$ obeys the system of ordinary differential equations

$$
\frac{\mathrm{d} N_{\alpha}}{\mathrm{d} t}=k_{\alpha}(\boldsymbol{N}) N_{\alpha}
$$

where $\boldsymbol{N}$ is vector with components $N_{\alpha}, k_{\alpha}(\boldsymbol{N})$ is the value of the reproduction coefficient $k_{\mu}$ at the point $x^{\alpha}$ :

$$
k_{\alpha}(\boldsymbol{N})=k_{\mu}\left(x^{\alpha}\right) \text { for } \mu=\sum_{\alpha} N_{\alpha} \delta\left(x-x^{\alpha}\right) .
$$

Let the dynamics of the system (3.21) for a given set of initial conditions be simple: the motion $\boldsymbol{N}(t)$ goes to the stable fixed point $\boldsymbol{N}=\boldsymbol{N}^{*}\left(\left\{x^{\alpha}\right\}\right)$. Then we can take in the right hand side of $(3.20)$

$$
\mu(t)=\mu^{*}\left(\left\{x^{\alpha}(t)\right\}\right)=\sum_{\alpha} N_{\alpha}^{*} \delta\left(x-x^{\alpha}(t)\right) .
$$

Because of the time separation we can assume that (i) relaxation of the amplitudes of peaks is completed and (ii) peaks are sufficiently narrow, hence, the difference between true $k_{\mu(t)}$ and the reproduction coefficient for the measure (3.22) with the finite support $\left\{x^{\alpha}\right\}$ is negligible. Let us use the notation $k^{*}\left(\left\{x^{\alpha}\right\}\right)(x)$ for this reproduction coefficient. The relations (3.20) transform into the ordinary differential equations

$$
\begin{aligned}
\sum_{j} q_{i j}^{\alpha} \frac{\mathrm{d} x_{j}^{\alpha}}{\mathrm{d} t} & =\left.\frac{\partial k^{*}\left(\left\{x^{\beta}\right\}\right)(x)}{\partial x_{i}}\right|_{x=x^{\alpha}} ; \\
\frac{\mathrm{d} q_{i j}^{\alpha}}{\mathrm{d} t} & =-\left.\frac{\partial^{2} k^{*}\left(\left\{x^{\beta}\right\}\right)(x)}{\partial x_{i} \partial x_{j}}\right|_{x=x^{\alpha}} .
\end{aligned}
$$

For many purposes it may be useful to switch to the logarithmic time $\tau=\ln t$ and to new variables

$$
b_{i j}^{\alpha}=\frac{1}{t} q_{i j}^{\alpha}=-\left.\frac{\partial^{2}\langle k(\mu)\rangle_{t}}{\partial x_{i} \partial x_{j}}\right|_{x=x^{\alpha}} .
$$

For large $t$ we obtain from (3.23)

$$
\begin{aligned}
\sum_{j} b_{i j}^{\alpha} \frac{\mathrm{d} x_{j}^{\alpha}}{\mathrm{d} \tau} & =\left.\frac{\partial k^{*}\left(\left\{x^{\beta}\right\}\right)(x)}{\partial x_{i}}\right|_{x=x^{\alpha}} ; \\
\frac{\mathrm{d} b_{i j}^{\alpha}}{\mathrm{d} \tau} & =-\left.\frac{\partial^{2} k^{*}\left(\left\{x^{\alpha}\right\}\right)(x)}{\partial x_{i} \partial x_{j}}\right|_{x=x^{\beta}}-b_{i j}^{\alpha} .
\end{aligned}
$$


The way of constructing the drift equations $(3.23,3.24)$ for a specific system (1.1) is as follows:

1. For finite sets $\left\{x^{\alpha}\right\}$ one studies systems (3.21) and finds the equilibrium solutions $\boldsymbol{N}^{*}\left(\left\{x^{\alpha}\right\}\right)$

2. For given measures $\mu^{*}\left(\left\{x^{\alpha}(t)\right\}\right)$ (3.22) one calculates the reproduction coefficients $k_{\mu}(x)=k^{*}\left(\left\{x^{\alpha}\right\}\right)(x)$ and first derivatives of these functions in $x$ at points $x^{\alpha}$. That is all, the drift equations $(3.23,3.24)$ are set up.

The drift equations $(3.23,3.24)$ describe the dynamics of the peaks positions $x^{\alpha}$ and of the coefficients $q_{i j}^{\alpha}$. For given $x^{\alpha}, q_{i j}^{\alpha}$ and $N_{\alpha}^{*}$ the distribution density $\mu$ can be approximated as the sum of narrow Gaussian peaks:

$$
\mu=\sum_{\alpha} N_{\alpha}^{*} \sqrt{\frac{\operatorname{det} Q^{\alpha}}{(2 \pi)^{n}}} \exp \left(-\frac{1}{2} \sum_{i j} q_{i j}^{\alpha}\left(x_{i}-x_{i}^{\alpha}\right)\left(x_{j}-x_{j}^{\alpha}\right)\right),
$$

where $Q^{\alpha}$ is the inverse covariance matrix $\left(q_{i j}^{\alpha}\right)$.

If the limit dynamics of the system (3.21) for finite supports at $t \rightarrow \infty$ can be described by a more complicated attractor, then instead of reproduction coefficient $k^{*}\left(\left\{x^{\alpha}\right\}\right)(x)=k_{\mu^{*}}$ for the stationary measures $\mu^{*}(3.22)$ one can use the average reproduction coefficient with respect to the corresponding Sinai-Ruelle-Bowen measure [43, 49]. If finite systems (3.21) have several attractors for given $\left\{x^{\alpha}\right\}$, then the dependence $k^{*}\left(\left\{x^{\alpha}\right\}\right)$ is multi-valued, and there may be bifurcations and hysteresis with the function $k^{*}\left(\left\{x^{\alpha}\right\}\right)$ transition from one sheet to another. There are many interesting effects concerning peaks' birth, desintegration, divergence, and death, and the drift equations $(3.23,3.24)$ describe the motion in a noncritical domain, between these critical effects.

Inheritance (conservation of support) is never absolutely exact. Small variations, mutations, immigration in biological systems are very important. Excitation of new degrees of freedom, modes diffusion, noise are present in physical systems. How does small perturbation in the inheritance affect the effects of selection? The answer is usually as follows: there is such a value of perturbation of the right-hand side of (1.1), at which they would change nearly nothing, just the limit $\delta$-shaped peaks transform into sufficiently narrow peaks, and zero limit of the velocity of their drift at $t \rightarrow \infty$ substitutes by a small finite one.

\subsection{Drift in Presence of Mutations and Scaling Invariance}

The simplest model for "inheritance + small variability" is given by a perturbation of (1.1) with diffusion term

$$
\frac{\partial \mu(x, t)}{\partial t}=k_{\mu(x, t)} \times \mu(x, t)+\varepsilon \sum_{i j} d_{i j}(x) \frac{\partial^{2} \mu(x, t)}{\partial x_{i} \partial x_{j}} .
$$

where $\varepsilon>0$ and the matrix of diffusion coefficients $d_{i j}$ is symmetric and positively definite. 
There are almost always no qualitative changes in the asymptotic behavior, if $\varepsilon$ is sufficiently small. With this the asymptotics is again described by the drift equations $(3.23,3.24)$, modified by taking into account the diffusion as follows:

$$
\begin{aligned}
\sum_{j} q_{i j}^{\alpha} \frac{\mathrm{d} x_{j}^{\alpha}}{\mathrm{d} t} & =\left.\frac{\partial k^{*}\left(\left\{x^{\beta}\right\}\right)(x)}{\partial x_{i}}\right|_{x=x^{\alpha}} ; \\
\frac{\mathrm{d} q_{i j}^{\alpha}}{\mathrm{d} t} & =-\left.\frac{\partial^{2} k^{*}\left(\left\{x^{\beta}\right\}\right)(x)}{\partial x_{i} \partial x_{j}}\right|_{x=x^{\alpha}}-2 \varepsilon \sum_{k l} q_{i k}^{\alpha} d_{k l}\left(x^{\alpha}\right) q_{l j}^{\alpha} .
\end{aligned}
$$

Now, as distinct from (3.23), the eigenvalues of the matrices $Q^{\alpha}=\left(q_{i j}^{\alpha}\right)$ cannot grow infinitely. This is prevented by the quadratic terms in the right-hand side of the second equation (3.27).

Dynamics of (3.27) does not depend on the value $\varepsilon>0$ qualitatively, because of the obvious scaling property. If $\varepsilon$ is multiplied by a positive number $\nu$, then, upon rescalling $t^{\prime}=\nu^{-1 / 2} t$ and $q_{i j}^{\alpha \prime}=\nu^{-1 / 2} q_{i j}^{\alpha}$, we have the same system again. Multiplying $\varepsilon>0$ by $\nu>0$ changes only peak's velocity values by a factor $\nu^{1 / 2}$, and their width by a factor $\nu^{1 / 4}$. The paths of peaks' motion do not change at this for the drift approximation (3.27) (but the applicability of this approximation may, of course, change).

\subsection{Three Main Types of Stability}

Stable steady-state solutions of equations of the form (1.1) may be only the sums of $\delta$ functions - this was already mentioned. There is a set of specific conditions of stability, determined by the form of equations.

Consider a stationary distribution for (1.1) with a finite support

$$
\mu^{*}(x)=\sum_{\alpha} N_{\alpha}^{*} \delta\left(x-x^{* \alpha}\right) .
$$

Steady state of $\mu^{*}$ means, that

$$
k_{\mu^{*}}\left(x^{* \alpha}\right)=0 \text { for all } \alpha .
$$

The internal stability means, that this distribution is stable with respect to perturbations not increasing the support of $\mu^{*}$. That is, the vector $N_{\alpha}^{*}$ is the stable fixed point for the dynamical system (3.21). Here, as usual, it is possible to distinguish between the Lyapunov stability, the asymptotic stability and the first approximation stability (negativeness of real parts for the eigenvalues of the matrix $\partial \dot{N}_{\alpha}^{*} / \partial N_{\alpha}^{*}$ at the stationary points).

The external stability (uninvadability) means stability to an expansion of the support, i.e. to adding to $\mu^{*}$ of a small distribution whose support contains points not belonging to $\operatorname{supp} \mu^{*}$. It makes sense to speak about the external stability only if there is internal stability. In this case it is sufficient to restrict ourselves with $\delta$-functional perturbations. The external stability has a very transparent physical and biological sense. It is stability with respect to introduction into the systems of a new inherited unit (gene, variety, specie...) in a small amount. 
The necessary condition for the external stability is: the points $\left\{x^{* \alpha}\right\}$ are points of the global maximum of the reproduction coefficient $k_{\mu^{*}}(x)$. It can be formulated as the optimality principle

$$
k_{\mu^{*}}(x) \leq 0 \text { for all } x ; k_{\mu^{*}}\left(x^{* \alpha}\right)=0 .
$$

The sufficient condition for the external stability is: the points $\left\{x^{* \alpha}\right\}$ and only these points are points of the global maximum of the reproduction coefficient $k_{\mu^{*}}\left(x^{* \alpha}\right)$. At the same time it is the condition of the external stability in the first approximation and the optimality principle

$$
k_{\mu^{*}}(x)<0 \text { for } x \notin\left\{x^{* \alpha}\right\} ; k_{\mu^{*}}\left(x^{* \alpha}\right)=0 .
$$

The only difference from (3.29) is the change of the inequality sign from $k_{\mu^{*}}(x) \leq 0$ to $k_{\mu^{*}}(x)<0$ for $x \notin\left\{x^{* \alpha}\right\}$. The necessary condition (3.29) means, that the small $\delta$-functional addition will not grow in the first approximation. According to the sufficient condition (3.30) such a small addition will exponentially decrease.

If $X$ is a finite set, then the combination of the external and the internal stability is equivalent to the standard stability for a system of ordinary differential equations.

For the continuous $X$ there is one more kind of stability important from the applications viewpoint. Substitute $\delta$-shaped peaks at the points $\left\{x^{* \alpha}\right\}$ by narrow Gaussians and shift slightly the positions of their maxima away from the points $x^{* \alpha}$. How will the distribution from such initial conditions evolve? If it tends to $\mu$ without getting too distant from this steady state distribution, then we can say that the third type of stability - stable realizability - takes place. It is worth mentioning that the perturbation of this type is only weakly* small, in contrast to perturbations considered in the theory of internal and external stability. Those perturbations are small by their norms. Let us remind that the norm of the measure $\mu$ is $\|\mu\|=\sup _{|f| \leq 1}[\mu, f]$. If one shifts the $\delta$-measure of unite mass by any nonzero distance $\varepsilon$, then the norm of the perturbation is 2 . Nevertheless, this perturbation weakly* tends to 0 with $\varepsilon \rightarrow 0$.

In order to formalize the condition of stable realizability it is convenient to use the drift equations in the form (3.24). Let the distribution $\mu^{*}$ be internally and externally stable in the first approximations. Let the points $x^{* \alpha}$ of global maxima of $k_{\mu^{*}}(x)$ be non-degenerate in the second approximation. This means that the matrices

$$
b_{i j}^{* \alpha}=-\left(\frac{\partial^{2} k_{\mu^{*}}(x)}{\partial x_{i} \partial x_{j}}\right)_{x=x^{* \alpha}}
$$

are strictly positively definite for all $\alpha$.

Under these conditions of stability and non-degeneracy the coefficients of (3.24) can be easily calculated using Taylor series expansion in powers of $\left(x^{\alpha}-x^{* \alpha}\right)$. The stable realizability of $\mu^{*}$ in the first approximation means that the fixed point of the drift equations (3.24) with the coordinates

$$
x^{\alpha}=x^{* \alpha}, b_{i j}^{\alpha}=b_{i j}^{* \alpha}
$$


is stable in the first approximation. It is the usual stability for the system (3.24) of ordinary differential equations, and these conditions with the notion of the stable realizability became clear from the logarithmic time drift equations (3.24) directly.

To explain the sense of the stable realizability we used in the book [32] the idea of the "Gardens of Eden" from J. H. Conway "Game of Life" [24]. That are Game of Life patterns which have no father patterns and therefore can occur only at generation 0 , from the very beginning. It is not known if a pattern which has a father pattern, but no grandfather pattern exists. It is the same situation, as for internal and external stable (uninvadable) state which is not stable realizable: it cannot be destroyed by mutants invasion and by the small variation of conditions, but, at the same time, it is not attractive for drift, and, hence, can not be realized in this asymptotic motion. It can be only created.

The idea of drift and the corresponding stability notions become necessary in any approach to evolutionary dynamics on continuous paces. In recent paper [21], the asymptotic stability under the replicator dynamics over a continuum of pure strategies was studied. It was shown in [21] that strong uninvadability of a pure strategy $x^{*}$ [4] is insufficient for its stability with respect to the drift: It does not imply convergence to $x^{*}$ when starting from a distribution of small deviations from $x^{*}$, regardless of how small these deviations are. The standard idea of asymptotic stability is: "after small deviation the system returns to the initial regime, and do not deviate to much on the way of returning". The crucial question for the measure dynamics is: in which topology the deviation is small? The small shift of the narrow peak of distribution in the continuous space of strategies can be considered as a small deviation in the weak* topology, but it is definitely large deviation in the strong topology, for example, if the shift is not small in comparison with the peak with. In the papers $[62,63]$ the idea of drift equations appeared again for the gaussian peaks in the dynamics of continuous symmetric evolutionary games. The authors $[62,63]$ introduced the idea of "evolutionary robustness" (realizability) and claimed the necessity of the additional notion of stability very energetically: "Furthermore, we provide new conditions for the stability of rest points and show that even strict equilibria may be unstable".

\section{Example: Cell Division Self-Synchronization}

The results described above admit for a whole family of generalizations. In particular, it seems to be important to extend the theorems of selection to the case of vector distributions, when $k_{\mu}(x)$ is a linear operator at each $\mu, x$. In this case, in the optimality principles for steady-states distributions the maximal eigenvalues of these operators $k_{\mu}(x)$ appear instead of the values of the reproduction coefficients. For general $\omega$-limit sets special multiplicative operator averages are in use [29]. It is possible also to make generalizations for some classes of non-autonomous equations with explicit dependencies of $k_{\mu}(x)$ on $t$ [29].

Availability of such a network of generalizations allows to construct the reasoning as follows: what is inherited (i.e. for what the law of conservation of support holds) is the subject of selection (i.e. with respect to these variables at $t \rightarrow \infty$ the distribution becomes 
discrete and the limit support can be described by the optimality principles).

This section gives a somewhat unconventional example of inheritance and selection, when the reproduction coefficients are subject additional conditions of symmetry.

Consider a culture of microorganisms in a certain medium (for example, pathogenous microbes in the organism of a host). Assume, for simplicity, the following: let the time period spent by these microorganisms for the whole life cycle be identical.

At the end of the life cycle the microorganism disappears and new several microorganisms appear in the initial phase. Let $T$ be the time of the life cycle. Each microorganism holds the value of the inherited variable, it is "the moment of its appearance $(\bmod T)$ ". Indeed, if the given microorganism emerges at time $\tau(0<\tau \leq T)$, then its first descendants appear at time $T+\tau$, the next generation - at the moment $2 T+\tau$, then $3 T+\tau$ and so on.

It is natural to assume that the phase $\tau(\bmod T)$ is the inherited variable with some accuracy. This implies selection of phases and, therefore, survival of their discrete number $\tau_{1}, \ldots \tau_{m}$, only. But results of the preceding sections cannot be applied directly to this problem. The reason is the additional symmetry of the system with respect to the phase shift. But the typicalness of selection and the instability of the uniform distribution over the phases $\tau(\bmod T)$ can be shown for this case, too. Let us demonstrate it with the simplest model.

Let the difference between the microorganisms at each time moment be related to the difference in the development phases only. Let us also assume that the state of the medium can be considered as a function of the distribution $\mu(\tau)$ of microorganisms over the phases $\tau \in] 0, T]$ (the quasi-steady state approximation for the medium). Consider the system at discrete times $n T$ and assume the coefficient connecting $\mu$ at moments $n T$ and $n T+T$ to be the exponent of the linear integral operator value:

$$
\mu_{n+1}(\tau)=\mu_{n}(\tau) \exp \left[k_{0}-\int_{0}^{T} k_{1}\left(\tau-\tau^{\prime}\right) \mu_{n}\left(\tau^{\prime}\right) \mathrm{d} \tau^{\prime}\right]
$$

Here, $\mu_{n}(\tau)$ is the distribution at the moment $n T, k_{0}=$ const, $k_{1}(\tau)$ is a periodic function of period $T$.

This model is constructed in order to study the interaction of two factors of microbial dynamics: the fixed period of the cell cycle, and the density-dependent interaction with the medium. The density-dependent interactions is modeled in the same degree of generality, as in general Volterra equations, with one addition: for systems with discrete time the exponential form of reproduction coefficient is more natural and useful, it was shown by Ricker [69]. In this sense, (4.33) presents a hybrid Volterra-Ricker model for microbial population with the fixed period of the cell cycle. The deviation from the fixed period can be formalized as a phase diffusion, as it was presented for the general systems with inheritance in the previous section, and here we study the "pure" consequences of phase inheritance.

This model significantly differs from the continuous time models, where the cell splitting is presented as a "quasi-chemical process" of fission of a cell with size parameter $2 x$ into two identical daughters with parameter $x:[2 x] \rightarrow[x]+[x]$ (any cell with size parameter $x$ can 
spontaneously split at any time without any dependence on its history). The probability of splitting depends here on the cell size only.

For example, a linear model for the growth of such a size-structured cell population, reproducing by continuous "Markov" fission of cells is formulated and identified in [18]. With known functions $\alpha(x)$ (death), $g(x)$ (growth) and $b(x)$ ("loss due to splitting") the model takes the form of a first order partial differential equation,

$$
\frac{\partial n(t, x)}{\partial t}+\frac{\partial(g n(t, x))}{\partial x}=-\alpha n(t, x)-b n(t, x)+4 b(2 x) n(t, 2 x),
$$

for the density $n(x, t)$. The equation is accompanied by proper initial and boundary conditions. With several restrictions it is proved that the asymptotic behavior of solutions for $t \rightarrow \infty$ has the form $n(t, x)=c e^{\lambda_{d} t}(\tilde{n}(x)+o(1))$, where the constant $c$ (single in the expression) depends on the initial distribution. The way to establish the sign of $\lambda_{d}$ and the explicit form of the function $\tilde{n}(x)$ is indicated. The continuous time Markov property (independence of history) with continuous kinetic coefficient $b(x)$ implies smoothing of limit distributions. In model (4.33) the cell remembers the moment of its "birth" and splits exactly after living time $T$. This property is the main difference between (4.33) and (4.34). The second difference is nonlinearity of (4.33), we take into account the cells density-dependent interaction (mediated by the medium state). The coefficient of this interaction, $k_{1}\left(\tau-\tau^{\prime}\right)$, depends on the age difference of interacting cells. This nonlinearity in (4.33) includes in implicit form the structure of population with age-determined size difference, etc.

The uniform steady-state $\mu^{*} \equiv n^{*}=$ const for (4.33) is:

$$
n^{*}=\frac{k_{0}}{\int_{0}^{T} k_{1}(\theta) \mathrm{d} \theta} .
$$

In order to examine stability of the uniform steady state $\mu^{*}(4.35)$, the system $(4.33)$ is linearized. For small deviations $\Delta \mu(\tau)$ in linear approximation

$$
\Delta \mu_{n+1}(\tau)=\Delta \mu_{n}(\tau)-n^{*} \int_{0}^{T} k_{1}\left(\tau-\tau^{\prime}\right) \Delta \mu_{n}\left(\tau^{\prime}\right) \mathrm{d} \tau^{\prime}
$$

Expand $k_{1}(\theta)$ into the Fourier series:

$$
k_{1}(\theta)=b_{0}+\sum_{n=1}^{\infty}\left(a_{n} \sin \left(2 \pi n \frac{\theta}{T}\right)+b_{n} \cos \left(2 \pi n \frac{\theta}{T}\right)\right) .
$$

Denote by $A$ operator of the right-hand side of (4.36). In the basis of functions

$$
e_{\mathrm{s} n}=\sin \left(2 \pi n \frac{\theta}{T}\right), e_{\mathrm{c} n}=\cos \left(2 \pi n \frac{\theta}{T}\right)
$$

on the segment $] 0, T]$ the operator $A$ is block-diagonal. The vector $e_{0}$ is eigenvector, $A e_{0}=$ $\lambda_{0} e_{0}, \lambda_{0}=1-n^{*} b_{0} T$. On the two-dimensional space, generated by vectors $e_{\mathrm{s} n}, e_{\mathrm{c} n}$ the 
operator $A$ is acting as a matrix

$$
A_{n}=\left(\begin{array}{cc}
1-\frac{T n^{*}}{2} b_{n} & -\frac{T n^{*}}{2} a_{n} \\
\frac{T n^{*}}{2} a_{n} & 1-\frac{T n^{*}}{2} b_{n}
\end{array}\right) .
$$

The corresponding eigenvalues are

$$
\lambda_{n 1,2}=1-\frac{T n^{*}}{2}\left(b_{n} \pm i a_{n}\right)
$$

For the uniform steady state $\mu^{*}(4.35)$ to be unstable it is sufficient that the absolute value of at least one eigenvalue $\lambda_{n 1,2}$ be larger than 1: $\left|\lambda_{n 1,2}\right|>1$. If there is at least one negative Fourier cosine-coefficient $b_{n}<0$, then $\operatorname{Re} \lambda_{n}>1$, and thus $\left|\lambda_{n}\right|>1$.

Note now, that almost all periodic functions (continuous, smooth, analytical - this does not matter) have negative Fourier cosine-coefficient. This can be understood as follows. The sequence $b_{n}$ tends to zero at $n \rightarrow \infty$. Therefore, if all $b_{n} \geq 0$, then, by changing $b_{n}$ at sufficiently large $n$, we can make $b_{n}$ negative, and the perturbation value can be chosen less than any previously set positive number. On the other hand, if some $b_{n}<0$, then this coefficient cannot be made non-negative by sufficiently small perturbations. Moreover, the set of functions that have all Fourier cosine-coefficient non-negative is completely thin, because for any compact of functions $K$ (for most of norms in use) the sequence $B_{n}=$ $\max _{f \in K}\left|b_{n}(f)\right|$ tends to zero, where $b_{n}(f)$ is the $n$th Fourier cosine-coefficient of function $f$.

The model (4.33) is revealing, because for it we can trace the dynamics over large times, if we restrict ourselves with a finite segment of the Fourier series for $k_{1}(\theta)$. Describe it for

$$
k_{1}(\theta)=b_{0}+a \sin \left(2 \pi \frac{\theta}{T}\right)+b \cos \left(2 \pi \frac{\theta}{T}\right) .
$$

Assume further that $b<0$ (then the homogeneous distribution $\mu^{*} \equiv \frac{k_{0}}{b_{0} T}$ is unstable) and $b_{0}>\sqrt{a^{2}+b^{2}}$ (then the $\int \mu(\tau) \mathrm{d} \tau$ cannot grow unbounded in time). Introduce notations

$$
\begin{aligned}
& M_{0}(\mu)=\int_{0}^{T} \mu(\tau) \mathrm{d} \tau, M_{\mathrm{c}}(\mu)=\int_{0}^{T} \cos \left(2 \pi \frac{\tau}{T}\right) \mu(\tau) \mathrm{d} \tau \\
& M_{\mathrm{s}}(\mu)=\int_{0}^{T} \sin \left(2 \pi \frac{\tau}{T}\right) \mu(\tau) \mathrm{d} \tau,\langle\mu\rangle_{n}=\frac{1}{n} \sum_{m=0}^{n-1} \mu_{m}
\end{aligned}
$$

where $\mu_{m}$ is the distribution $\mu$ at the discrete time $m$.

In these notations,

$$
\begin{aligned}
\mu_{n+1}(\tau)= & \mu_{n}(\tau) \exp \left[k_{0}-b_{0} M_{0}\left(\mu_{n}\right)-\left(a M_{\mathrm{c}}\left(\mu_{n}\right)+b M_{\mathrm{s}}\left(\mu_{n}\right)\right) \sin \left(2 \pi \frac{\tau}{T}\right)\right. \\
& \left.+\left(a M_{\mathrm{s}}\left(\mu_{n}\right)-b M_{\mathrm{c}}\left(\mu_{n}\right)\right) \cos \left(2 \pi \frac{\tau}{T}\right)\right] .
\end{aligned}
$$


Represent the distribution $\mu_{n}(\tau)$ through the initial distribution $\mu_{0}(\tau)$ and the functionals $M_{0}, M_{\mathrm{c}}, M_{\mathrm{s}}$ values for the average distribution $\left.\langle\mu\rangle_{n}\right)$ :

$$
\begin{aligned}
\mu_{n}(\tau)= & \mu_{0}(\tau) \\
& \times \exp \left\{n \left[k_{0}-b_{0} M_{0}\left(\langle\mu\rangle_{n}\right)-\left(a M_{\mathrm{c}}\left(\langle\mu\rangle_{n}\right)+b M_{\mathrm{s}}\left(\langle\mu\rangle_{n}\right)\right) \sin \left(2 \pi \frac{\tau}{T}\right)\right.\right. \\
& \left.\left.+\left(a M_{\mathrm{s}}\left(\langle\mu\rangle_{n}\right)-b M_{\mathrm{c}}\left(\langle\mu\rangle_{n}\right)\right) \cos \left(2 \pi \frac{\tau}{T}\right)\right]\right\} .
\end{aligned}
$$

The exponent in (4.41) is either independent of $\tau$, or there is a function with the single maximum on $] 0, T]$. The coordinate $\tau_{n}^{\#}$ of this maximum is easily calculated

$$
\tau_{n}^{\#}=-\frac{T}{2 \pi} \arctan \frac{a M_{\mathrm{c}}\left(\langle\mu\rangle_{n}\right)+b M_{\mathrm{s}}\left(\langle\mu\rangle_{n}\right)}{a M_{\mathrm{s}}\left(\langle\mu\rangle_{n}\right)-b M_{\mathrm{c}}\left(\langle\mu\rangle_{n}\right)}
$$

Let the non-uniform smooth initial distribution $\mu_{0}$ has the whole segment $\left.] 0, T\right]$ as its support. At the time progress the distributions $\mu_{n}(\tau)$ takes the shape of ever narrowing peak. With high accuracy at large $a$ we can approximate $\mu_{n}(\tau)$ by the Gaussian distribution (approximation accuracy is understood in the weak* sense, as closeness of mean values):

$$
\begin{aligned}
& \mu_{n}(\tau) \approx M_{0} \sqrt{\frac{q_{n}}{\pi}} \exp \left[-q_{n}\left(\tau-\tau_{n}^{\#}\right)^{2}\right], M_{0}=\frac{k_{0}}{k_{1}(0)}=\frac{k_{0}}{b_{0}+b}, \\
& q_{n}^{2}=n^{2}\left(\frac{2 \pi}{T}\right)^{4} \\
& \quad \times\left[\left(a M_{\mathrm{c}}\left(\langle\mu\rangle_{n}\right)+b M_{\mathrm{s}}\left(\langle\mu\rangle_{n}\right)\right)^{2}+\left(a M_{\mathrm{s}}\left(\langle\mu\rangle_{n}\right)-b M_{\mathrm{c}}\left(\langle\mu\rangle_{n}\right)\right)^{2}\right] .
\end{aligned}
$$

Expression (4.43) involves the average measure $\langle\mu\rangle_{n}$ which is difficult to compute. However, we can operate without direct computation of $\langle\mu\rangle_{n}$. At $q_{n} \gg \frac{1}{T^{2}}$ we can compute $q_{n+1}$ and $\tau_{n+1}^{\#}$ :

$$
\begin{aligned}
& \mu_{n+1} \approx M_{0} \sqrt{\frac{q_{n}+\Delta q}{\pi}} \exp \left[-\left(q_{n}+\Delta q\right)\left(\left(\tau-\tau_{n}^{\#}-\Delta \tau^{\#}\right)^{2}\right],\right. \\
& \Delta q \approx-\frac{1}{2} b M_{0}\left(\frac{2 \pi^{2}}{T}\right), \Delta \tau^{\#} \approx \frac{1}{q} M_{0} \frac{2 \pi}{T} .
\end{aligned}
$$

The accuracy of these expression grows with time $n$. The value $q_{n}$ grows at large $n$ almost linearly, and $\tau_{n}^{\#}$, respectively, as the sum of the harmonic series $(\bmod T)$, i.e. $\operatorname{as} \ln n(\bmod T)$. The drift effect takes place: location of the peak $\tau_{n}^{\#}$, passes at $n \rightarrow \infty$ the distance diverging as $\ln n$.

Of interest is the case, when $b>0$ but

$$
\left|\lambda_{1}\right|^{2}=\left(1+n^{*} b \frac{T}{2}\right)^{2}+\left(n^{*} a \frac{T}{2}\right)^{2}>1 .
$$




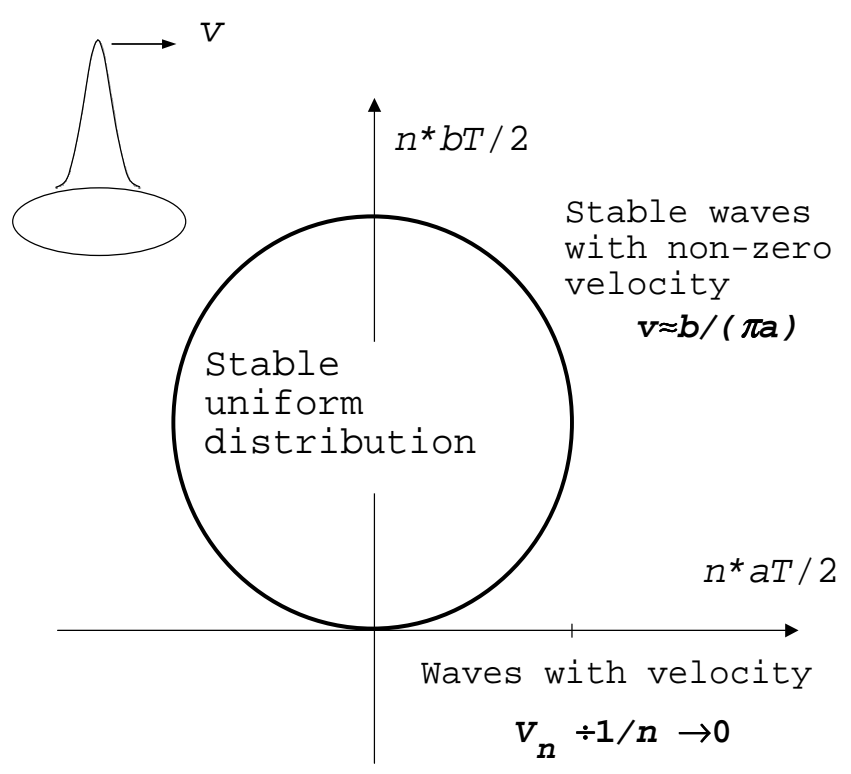

Figure 1: The simplest model of cell division self-synchronization: The parametric portrait.

With this, homogeneous distribution $\mu^{*} \equiv n^{*}$ is not stable but $\mu$ does not tend to $\delta$-functions. There are smooth stable "self-synchronization waves" of the form

$$
\mu_{n}=\gamma \exp \left[q \cos \left(\left(\tau-n \Delta \tau^{\#}\right) \frac{2 \pi}{T}\right)\right]
$$

At small $b>0\left(b \ll|a|, b M_{0} \ll a^{2}\right)$ we can find explicit form of approximated expressions for $q$ and $\Delta \tau^{\#}$ :

$$
q \approx \frac{a^{2} M_{0}}{2 b}, \Delta \tau^{\#} \approx \frac{b T}{\pi a}
$$

At $b>0, b \rightarrow 0$, smooth self-synchronization waves become ever narrowing peaks, and their steady velocity approaches zero. If $b=0,\left|\lambda_{1}\right|^{2}>1$, then the effect of selection takes place again, and for almost all initial conditions $\mu_{0}$ with the support being the whole segment ] $0, T]$ the distribution $\mu_{n}$ takes at large $n$ the form of a slowly drifting almost Gaussian peak. It becomes narrower with the time, and the motion slows down. Instead of the linear growth of $q_{n}$ which takes place at $b<0(4.44)$, for $b=0, q_{n+1}-q_{n} \approx \operatorname{const} q_{n}^{-1}$ and $q_{n}$ grows as const $\sqrt{n}$.

The parametric portrait of the system for the simple reproduction coefficient (4.40) is presented in Fig. 1.

As it is usual, a small desynchronization transforms $\delta$-functional limit peaks to narrow Gaussian peaks, and the velocity of peaks tends to small but nonzero velocity instead of zero. The systems with small desynchronization can be described by equations of the form (3.27). The large desynchronization can completely destroy the effects of phase selection and, for example, it might lead to the globally stable uniform phase distribution. 
There are many specific mechanisms of synchronization and desynchronysation in physics and biology (see, for example, $[3,64,47,61,13]$ ). We described here very simple, but universal mechanism: it requires only that the time of the life cycle is fixed, in this case in a generic situation we should observe the self-synchronization. Of course, the real-world situation can be much more complicated, with a plenty of additional factors, but the basic mechanism of the "phase selection" works always, if the life cycle has more or less fixed duration.

\section{Conclusion: Main Results About Systems With Inheritance}

1. If a kinetic equation has the quasi-biological form (1.1), then it has a rich system of invariant manifolds: for any closed subset $A \subset X$ the set of distributions $\boldsymbol{M}_{A}=\{\mu \mid$ $\operatorname{supp} \mu \subseteq A\}$ is invariant with respect to the system (1.1). These invariant manifolds form important algebraic structure, the summation of manifolds is possible:

$$
M_{A} \oplus M_{B}=M_{A \cup B} .
$$

(Of course, $\boldsymbol{M}_{A \cap B}=\boldsymbol{M}_{A} \cap \boldsymbol{M}_{B}$ ).

2. Typically, all the $\omega$-limit points belong to invariant manifolds $\boldsymbol{M}_{A}$ with finite $A$ (from the application point of view there is no difference between finite and almost finite sets). The finite-dimensional approximations of the reproduction coefficient (2.11) provides the minimax estimation of the number of points in $A$.

3. Typically, systems with inheritance have a rich family of Lyapunov functionals of form (2.19) similar to the Burg entropy, these functionals can be interpreted as the measures of decreasing diversity.

4. For systems with inheritance (1.1) a solution typically tends to be a finite set of narrow peaks getting in time more and more narrow, moving slower and slower. It is possible that these peaks do not tend to fixed positions, rather they continue moving, and the path covered tends to infinity at $t \rightarrow \infty$. This is the drift effect.

5. The equations for peak dynamics, the drift equations, $(3.23,3.24,3.27)$ describe dynamics of the shapes of the peaks and their positions. For systems with small variability ("mutations") the drift equations (3.27) has the scaling property: the change of the intensity of mutations is equivalent to the change of the time scale.

6. Three specific types of stability are important for the systems with inheritance: internal stability (stability with respect to perturbations without extension of distribution support), external stability (stability with respect to small one-point extension of distribution support), and stable realizability (stability with respect to weakly* small 
perturbations: small extensions and small shifts of the peaks; these perturbations are small in the weak* topology.).

The cell division self-synchronization demonstrates effects of unusual inherited unit, it is an example of a "phase selection". One specific property of this selection is additional symmetry with respect to phase shift. In this case, the general results about selection cannot be used directly. Nevertheless, the "equivariant" selection theory successfully works too.

Some exact results of the mathematical selection theory can be found in $[51,52]$. There exist many physical examples of systems with inheritance [86, 87, 55, 23, 50, 84, 74]. A wide field of ecological applications was described in the book [73]. An introduction into adaptive dynamics was given in notes [16] that illustrate largely by way of examples, how standard ecological models can be put into an evolutionary perspective in order to gain insight in the role of natural selection in shaping life history characteristics.

Acknowledgements. Author is grateful to V. Okhonin, who involved him many years ago in the analysis of mathematical models of natural selection. M. Gromov kindly allowed to quote his talk [38].

\section{References}

[1] G. D. Birkhoff. Dynamical systems. AMS Colloquium Publications, Providence, 1927.

[2] D. T. Bishop, C. Cannings. A generalized war of attrition. J. Theor. Biol. 70 (1978), 85-124.

[3] I. I. Blekhman. Synchronization in science and technology. ASME Press, New York, 1988.

[4] I. M. Bomze. Dynamical aspects of evolutionary stability. Monatshefte für Mathematik 110 (1990), 189-206.

[5] I. M. Bomze. Detecting all evolutionarily stable strategies. J. Optim. Theory Appl. 75 (1992), 313-329.

[6] I. M. Bomze. Uniform barriers and evolutionarily stable sets. In: Game theory, experience, rationality. Foundations of social sciences, economics and ethics. In honor of John C. Harsanyi (W. Leinfellner, E. Köhler, eds.). Vienna Circle Institute Yearbook, 5. Kluwer Academic Publishers, Dordrecht, 1998, pp. 225-243.

[7] I. M. Bomze. Regularity vs. degeneracy in dynamics, games, and optimization: a unified approach to different aspects. SIAM Review 44 (2002), 394-414.

[8] I. M. Bomze, R. Bürger. Stability by mutation in evolutionary games, evolutionary game theory in biology and economics. Games Econom. Behav. 11(2) (1995), 146-172. 
[9] I. M. Bomze, B. M. Pötscher. Game Theoretical Foundations of Evolutionary Stability. Lecture Notes in Economics and Math. Systems 324, Springer, Berlin, 1989.

[10] N. Bourbaki. Elements of mathematics - Integration I. Springer, Berlin, 2003.

[11] J. P. Burg. The Relationship Between Maximum Entropy Spectra and Maximum Likelihood Spectra. Geophysics 37 (1972), 375-376.

[12] J. H. Conway, R. K. Guy. Liouville's Number. In: J. H. Conway, R. K. Guy. The Book of Numbers. Springer, New York, 1996, pp. 239-241.

[13] S. Cooper. Minimally disturbed, multi-cycle, and reproducible synchrony using a eukaryotic 'baby machine'. Bioessays 24 (2002), 499-501.

[14] R. Cressman. The Stability Concept of Evolutionary Game Theory. Lecture Notes in Biomathematics 94. Springer, Berlin, 1992.

[15] Ch. Darwin. On the origin of species by means of natural selection, or preservation of favoured races in the struggle for life. A Facsimile of the First Edition. Harvard University Press, Cambridge MA, 1964.

[16] O. Diekmann. A beginner's guide to adaptive dynamics. In: Mathematical modelling of population dynamics. Banach Center Publications, Vol. 63. Institute of Mathematics Polish Academy of Sciences, Warszawa, 2004, pp. 47-86.

[17] O. Diekmann, M. Gyllenberg, J. A. J. Metz. Steady state analysis of structured population models. Theor. Popul. Biol. 63 (2003), 309-338.

[18] O. Diekmann, H. J. Heijmans, H. R. Thieme. On the stability of the cell size distribution. J. Math. Biol. 19 (1984), 227-248.

[19] M. Doebeli, U. Dieckmann. Speciation along environmental gradient. Nature 421 (2003), 259-264.

[20] Y. E. Dubrova. Radiation-induced transgenerational instability. Oncogene 22(45) (2003), 7087-7093.

[21] I. Eshel, E. Sansone. Evolutionary and dynamic stability in continuous population games. J. Math. Biology 46 (2003), 445-459.

[22] W. J. Ewens. Mathematical Population Genetics. Springer, Berlin, 1979.

[23] A. B. Ezersky,M. I. Rabinovich. Nonlinear-wave competition and anisotropic spectra of spatiotemporal chaos of Faraday ripples. Europhys. Lett. 13 (1990), 243-249.

[24] M. Gardner. On cellular automata, self-reproduction, the garden of Eden and the game of 'life'. Scientific American 224(2) (1971), 112-115. 
[25] M. B. Gerstein, C. Bruce, J. S. Rozowsky, D. Zheng, J. Du, J. O. Korbel, O. Emanuelsson, Z. D. Zhang, Sh. Weissman, M. Snyder. What is a gene, post-ENCODE? History and updated definition. Genome Res. 17 (2007), 669-681.

[26] G. F. Gause. The struggle for existence. Williams and Wilkins, Baltimore, 1934. (Available online: http://www.ggause.com/Contgau.htm)

[27] A. N. Gorban. Dynamical systems with inheritance. In: Some problems of community dynamics (R. G. Khlebopros, ed.). Institute of Physics RAS, Siberian Branch, Krasnoyarsk, 1980. [in Russian]

[28] A. N. Gorban. Systems with inheritance: dynamics of distributions with conservation of support, natural selection and finite-dimensional asymptotics. E-print, arXiv:condmat/0405451, 2004. (Available online: http://arxiv.org/abs/cond-mat/0405451)

[29] A. N. Gorban. Equilibrium encircling. Equations of chemical kinetics and their thermodynamic analysis. Nauka, Novosibirsk, 1984. [in Russian]

[30] A. N. Gorban. Order-disorder separation: Geometric revision, Physica A 374 (2007), 85-102.

[31] A. N. Gorban, I. V. Karlin. Family of additive entropy functions out of thermodynamic limit. Phys. Rev. E 67 (2003), 016104.

[32] A. N. Gorban, R. G. Khlebopros. Demon of Darwin: Idea of optimality and natural selection. Nauka (FizMatGiz), Moscow, 1988. [in Russian]

[33] A. N. Gorban, V. T. Manchuk, A. V. Perfil'eva, E. V. Smirnova, E. P. Cheusova. The mechanism of correlations increase for physiological parameters under adaptation stress, Sibirskii Ekologicheskii Zhurnal [Siberian Ecological Journal, Novosibirsk] 5 (2001), 651655.

[34] A. N. Gorban, V. T. Manchuk, E. V. Petushkova. Dynamics of physiological paramethers correlations and the ecological-evolutionary principle of polyfactoriality, Problemy Ekologicheskogo Monitoringa i Modelirovaniya Ekosistem [The Problems of Ecological Monitoring and Ecosystem Modelling], Vol. 10. Gidrometeoizdat, Leningrad, 1987, pp. 187-198.

[35] A. N. Gorban, M. G. Sadovskii. Optimal strategies of spatial distribution: the Allee effect. Zhurn. Obshch. Biol. 50 (1989), 16-21.

[36] A. N. Gorban, M. G. Sadovskii. Evolutionary mechanisms of creation of cellular clusters in flowrate Cultivators. Biotechnology and Biotechnics (Sofia) 2(5) (1987), 34-36.

[37] M. Grmela. Reciprocity relations in thermodynamics. Physica A 309 (2002), 304-328. 
[38] M. Gromov. A dynamical model for synchronisation and for inheritance in microevolution: a survey of papers of A.Gorban. The talk given in the IHES seminar, "Initiation to functional genomics: biological, mathematical and algorithmical aspects". Institut Henri Poincaré, November 16, 2000.

[39] J. B. S. Haldane. The Causes of Evolution. Princeton Science Library, Princeton University Press, Princeton, 1990.

[40] H. Haken. Synergetics, an introduction. Nonequilibrium phase transitions and selforganization in physics, chemistry and biology. Springer, Berlin, 1978.

[41] P. Hammerstein. Darwinian adaptation, population genetics and the streetcar theory of evolution. J. Math. Biol. 34 (1996), 511-532.

[42] G. Hardin. The Competitive Exclusion Principle. Science (1960), 29 April, 1292-1297.

[43] B. Hasselblatt, A. Katok (Eds.). Handbook of Dynamical Systems. Vol. 1A. Elsevier, Amsterdam, 2002.

[44] J. Hofbauer, P. Schuster, K. Sigmund. A note on evolutionary stable strategies and game dynamics. J. Theor. Biol. 81 (1979), 609-612.

[45] J. Hofbauer, K. Sigmund. Evolutionary game dynamics. Bull. (New Series) American Math. Soc. 40(4) (2003), 479-519.

[46] Y. Huang, O. Diekmann. Predator migration in response to prey density: what are the consequences? J. Math. Biol. 43 (2001), 561-581.

[47] K. Josić. Synchronization of chaotic systems and invariant manifolds. Nonlinearity 13 (2000), 1321-1336.

[48] I. V. Karmanova, V. N. Razzhevaikin, M. I. Shpitonkov, Application of correlation adaptometry for estimating a response of herbaceous species to stressial loadings. Doklady Akademii Nauk 346 (1996), 424-426.

[49] A. Katok, B. Hasselblat. Introduction to the Modern Theory of Dynamical Systems. Encyclopedia of Math. and its Applications, Vol. 54. Cambridge University Press, Cambridge, 1995.

[50] A. Krawiecki, A. Sukiennicki. Marginal synchronization of spin-wave amplitudes in a model for chaos in parallel pumping. Physica Status Solidi B 236 (2003), 511-514.

[51] O. A. Kuzenkov. Weak solutions of the Cauchy problem in the set of Radon probability measures. Differential Equations 36 (2000), 1676-1684.

[52] O. A. Kuzenkov. A dynamical system on the set of Radon probability measures. Differential Equations 31 (1995), 549-554. 
[53] S. M. Levin. Community equilibria and stability, and an extension of the competitive exclusion principle. Am. Nat. 104 (1970), 413423.

[54] L. A. Levin. Randomness conservation inequalities; Information and independence in mathematical theories. Information and Control 61 (1984), 15-37.

[55] V. S. L'vov. Wave turbulence under parametric excitation applications to magnets. Springer, Berlin, 1994.

[56] R. MacArthur, R. Levins. Competition, habitat selection, and character displacement in a patchy environment. Proc. Nat. Acad. Sci. 51 (1964), 1207-1210.

[57] R. MacArthur, R. Levins. The limiting similarity, convergence, and divergence of coexisting species. Am. Nat. 101 (1967), 377-385.

[58] E. Mayr. Animal Species and Evolution. Harvard University Press, Cambridge MA, 1963.

[59] G. Meszena, M. Gyllenberg, L. Pasztor, J. A. J. Metz. Competitive exclusion and limiting similarity: A unified theory. Theoretical Population Biology 69 (2006), 68-87.

[60] G. Meszena and J. A. J. Metz. Species diversity and population regulation: the importance of environmental feedback dimensionality. International Institute for Applied Systems Analysis, Interim Report IR-99-045, 1999.

[61] E. Mosekilde, Yu. Maistrenko, D. Postnov. Chaotic synchronization: Applications to living systems. World Scientific, Singapore, 2002.

[62] J. Oechssler, F. Riedel. Evolutionary dynamics on infinite strategy spaces. Economic Theory 17 (2001), 141-162.

[63] J. Oechssler, F. Riedel. On the Dynamic Foundation of Evolutionary Stability in Continuous Models. Journal of Economic Theory 107 (2002), 223-252.

[64] A. Pikovsky, M. Rosenblum, J. Kurths. Synchronization: A Universal Concept in Nonlinear Science. Cambridge University Press, Cambridge, 2002.

[65] L. I. Pokidysheva, R. A. Belousova, E. V. Smirnova. Method of adaptometry in the evaluation of gastric secretory function in children under conditions of the North. Vestn Ross. Akad. Med. Nauk (1996), No. 5, 42-45.

[66] P. Racsko, M. Semenov. Analysis of mathematical principles in crop growth simulationmodels. Ecological Modelling 47 (1989), 291-302.

[67] O. J. Rando, K. J. Verstrepen. Timescales of Genetic and Epigenetic Inheritance. Cell 128 (2007), 655-668. 
[68] K. Räsänen, L. E. B. Kruuk. Maternal effects and evolution at ecological time-scales. Funct. Ecology 21 (2007), 408-421.

[69] W. Ricker. Stock and recruitment. J. Fish. Res. Board Canada 11 (1954), 559-663.

[70] L. I. Rozonoer, E. I. Sedyh. On the mechanisms of of evolution of self-reproduction systems. 1, 2, 3. Automation and Remote Control 40 (1979), 243-251, 419-429, 741749 .

[71] M. G. Sadovskii. 'Prey-predator' model with purposeful space migration of individuals. Zhurn. Obshch. Biol. 62(3) (2001), 239-245.

[72] K. R. Sedov, A. N. Gorban, E. V. Petushkova, V. T. Manchuk, E. N. Shalamova. Correlation adaptometry as a method of screening of the population. Vestn. Akad. Med. Nauk SSSR (1988), No. 10, 69-75.

[73] F. N. Semevsky, S. M. Semenov. Mathematical modeling of ecological processes. Gidrometeoizdat, Leningrad, 1982 [in Russian]

[74] V. P. Seminozhenko. Kinetics of interacting quasiparticles in strong external fields. Phys. Reports 91(3) (1982), 103-182.

[75] V. T. Sergovantsev. Structures and properties of control-systems for evolution in nature and society, Automation and Remote Control 54 (1993), 323-331.

[76] J. M. Smith, G. R. Price. The Logic of Animal Conflict. Nature 246 (1973), 15-18.

[77] J. M. Smith. Evolution and the theory of games. Cambridge University Press, Cambridge, 1982.

[78] G. N. Svetlichnaia, E. V. Smirnova, L. I. Pokidysheva. Correlational adaptometry as a method for evaluating cardiovascular and respiratory interaction. Fiziol Cheloveka [Human Physiology, Moscow] 23(3) (1997) 58-62.

[79] P. Taylor. Evolutionary stable strategies and game dynamics. J. Appl. Probability 16 (1979), 76-83.

[80] B. Thomas. On evolutionarily stable sets. J. Math. Biol. 22 (1985), 105-115.

[81] T. J. M. Van Dooren, J. A. J. Metz. Delayed maturation in temporally structured populations with non-equilibrium dynamics. J. Evol. Biol. 11 (1998), 41-62.

[82] G. T. Vickers, C. Cannings. Patterns of ESSs. I, II. J. Theoret. Biol. 132(4) (1988), 387-408, 409-420.

[83] V. Volterra. Lecons sur la théorie mathematique de la lutte pour la vie. Gauthier-Villars, Paris, 1931. 
[84] V. M. Vorobev. Selection of normal variables for unstable conservative media. Zhurnal Tekhnicheskoi Fiziki 62(8) (1992), 172-175.

[85] G. S. Yablonskii, V. I. Bykov, A. N. Gorban, V. I. Elokhin. Kinetic models of catalytic reactions. Comprehensive Chemical Kinetics, Vol. 32 (R. G. Compton, ed.). Elsevier, Amsterdam, 1991.

[86] V. E. Zakharov, V. S. L'vov, S. S. Starobinets. Turbulence of spin-waves beyond threshold of their parametric-excitation. Uspekhi Fizicheskikh Nauk 114(4) (1974), 609-654. [English translation: Soviet Physics - Uspekhi 17(6) (1975), 896-919.]

[87] V. E. Zakharov, V. S. L'vov, G. E. Falkovich. Kolmogorov spectra of turbulence. Vol. 1. Wave Turbulence. Springer, Berlin, 1992. 